\title{
THE SYMPLECTIC FLOER HOMOLOGY OF COMPOSITE KNOTS
}

\author{
WEIPING LI
}

\begin{abstract}
We develop a method of calculation for the symplectic Floer homology of composite knots. The symplectic Floer homology of knots defined in 15] naturally admits an integer graded lifting, and it formulates a filtration and induced spectral sequence. Such a spectral sequence converges to the symplectic homology of knots in 15. We show that there is another spectral sequence which converges to the $\mathbb{Z}$-graded symplectic Floer homology for composite knots represented by braids.
\end{abstract}

\section{INTRODUCTION}

For integral homology 3-spheres $Y$, Casson defined an integral invariant which roughly counts the number of irreducible $S U(2)$ representations of the fundamental group $\pi_{1}(Y)$; Floer developed a $\mathbb{Z}_{8}$-graded instanton homology theory based upon an application of Morse theory to the Chern-Simons functional on the space $\mathcal{B}_{Y}$ of equivalence classes of $S U(2)$ connections on $Y$ (see [7]). The Euler characteristic of the instanton Floer homology is twice Casson's invariant. Lin [20] studied the special representations of knot group $\pi_{1}\left(S^{3} \backslash K\right)$ into $S U(2)$ such that all meridians of knot $K$ are represented by trace-zero matrices, and defined an invariant $\lambda_{C L}(K)$ for the knot $K$. The present author [15] developed a symplectic Floer homology based upon the Atiyah conjecture that there is no difference between the instanton Floer homology and the symplectic Floer homology for the integral homology 3 -spheres. Our symplectic Floer homology generalizes the invariant of Lin, and its Euler characteristic is the negative of $\lambda_{C L}(K)$. For $S U(2)$ representations of $\pi_{1}\left(S^{3} \backslash K\right)$ with the trace of all meridians fixed (not necessary zero), Cappell, Lee and Miller [3], independently Herald [12], defined the equivariant knot signature from the symplectic theory and the gauge theory points of view; the present author extended the symplectic Floer homology in 15] to the general case which the Euler characteristic is the equivariant knot signature in [17.

In this paper, we study the Mayer-Vietoris principle for the symplectic Floer homology defined in 15, 17]. We only restrict to the trace-zero case in 15 (the general case in 17] is similar). The natural operation among knots is the connected sum which is well-defined for the equivalence classes of knots. The sum operation is commutative and associative.

Date: February 2, 1998.

1991 Mathematics Subject Classification. Primary 57M25, 58F05; Secondary 57M05, 70H05.

Key words and phrases. Braid, Signature of a Knot, Symplectic Floer Homology.

Partially supported by NSF Grant DMS 9626166. 
Denote $K=K_{1} \# K_{2}$ for the connected sum of knots or the composite knot of $K_{1}$ and $K_{2}$. It turns out that there is a nice algebraic topology method to compute the symplectic Floer homology of the composite knot in terms of the ones of $K_{1}$ and $K_{2}$. The method we used in this paper is in principle the same for the instanton Floer homology [16, 18].

It is easy to see that the special representations of composite knot consists of two different types: a single special representation arising from one knot and the unique reducible special representation from the other, a circle of special representations arising from irreducible special representations of both knots (see Proposition 3.1). The circle can also be interpreted as a gluing parameters for two irreducible flat connections of the knot complement in [12]. We show that such a circle is a nondegenerate critical submanifold of the symplectic action in the Bott sense [2]. A natural algebraic topology method is to filter the critical submanifolds and to form a spectral sequence. This can not be done directly to our symplectic Floer homology in [15, 17] since the symplectic Floer homology of knots is $\mathbb{Z}_{2 N}$-graded in general ( $N=N(K)$ is not necessary zero). To overcome this difficulty, we define a $\mathbb{Z}$-graded symplectic Floer homology of the braid which is an integral lifting of the one in [15] (see [9]). Using the monotonicity and some properties of the special representation variety, we show that there is a well-defined $\mathbb{Z}$-graded symplectic Floer homology of braids. The $\mathbb{Z}$ graded symplectic Floer homology is only invariant under the Markov move of type I and its inverse (Proposition 2.11), not invariant under the Markov move of type II and its inverse. But there exists a nice relation between the $\mathbb{Z}$-graded and $\mathbb{Z}_{2 N}$-graded symplectic Floer homologies. One of our main theorems is the following.

\section{Theorem A:}

1. There is a spectral sequence $\left(E_{n, j}^{k}\left(\phi_{\beta}\right), d^{k}\right)$ for $K=\bar{\beta}$ with the $E_{n, j}^{1}\left(\phi_{\beta}\right)$ term given by the $\mathbb{Z}$-graded symplectic Floer homology of the braid representing the knot $K$.

2. The spectral sequence $\left(E_{n, j}^{k}\left(\phi_{\beta}\right), d^{k}\right)$ converges to the $\mathbb{Z}_{2 N}$-graded symplectic Floer homology of the knot $K=\bar{\beta}$.

Then we formulate a well-defined filtration for the $\mathbb{Z}$-graded symplectic Floer chain complex of the braid representing the composite knot via the critical submanifolds. The filtration for the $\mathbb{Z}$-graded chain complex of the composite knot derives another spectral sequence. Such a spectral sequence converges to the $\mathbb{Z}$-graded symplectic Floer homology of the composite knot. Using Theorem A, we obtain the $\mathbb{Z}_{2 N}$-graded symplectic Floer homology of the composite knot.

\section{Theorem B:}

1. There is a spectral sequence $\left(E_{p, q}^{r}\left(\phi_{\beta}\right), d_{r}\right)$ determined by the filtration 18$)$ for the braid $\beta=\beta_{1} \Sigma^{n-1}\left(\beta_{2}\right)$ of the composite knot $K_{1} \# K_{2}$.

2. The spectral sequence $\left(E_{p, q}^{r}, d_{r}\right)$ collapses at the third term. Thus $E_{*, *}^{3}=E_{*, *}^{\infty}$ gives the $\mathbb{Z}$-graded Floer homology $I_{*}^{\left(r_{1}, r_{2}\right)}\left(C_{*}^{\left(r_{1}, r_{2}\right)}\left(\beta_{1} \Sigma^{n-1}\left(\beta_{2}\right)\right)\right)$ of the braid $\beta$. 
By Theorem B, there are only two differentials needed to identify in order to compute the $\mathbb{Z}$-graded symplectic Floer homology of the braid representing the composite knot. The differential $d_{1}$ basically consists of the integral differential on each braid $\beta_{j}(j=1,2)$ and plus two special boundary maps contributed from/to the unique reducible special representation of $K_{j}(j=1,2)$. This is done by a cobordism argument for the moduli spaces of $J$-holomorphic curves with variations of Hamiltonian functions. We do not know how to characterize the differential $d_{2}$ at this moment.

Theorem C: The differential $d_{1}$ of the spectral sequence $\left(E_{p, q}^{r}\left(\phi_{\beta}\right), d_{r}\right)$ in Theorem $B$ is given by

$$
d_{1}=\partial_{1}^{\left(r_{1}\right)} \star I d_{2} \pm I d_{1} \star \partial_{2}^{\left(r_{2}\right)} \pm d_{K_{1}} \star I d_{2} \pm I d_{1} \star d_{K_{2}}+\delta_{K_{1}} \star I d_{2} \pm I d_{1} \star \delta_{K_{2}} .
$$

See Definition 4.1 for the two special boundary maps $d_{K_{j}}$ and $\delta_{K_{j}}(j=1,2)$.

This paper is organized as follows. In $\S 2.1$, we briefly review the symplectic Floer homology of knots, and extend the $\mathbb{Z}_{2 N}$-graded theory of knots to the $\mathbb{Z}$-graded theory of braids in $\S 2.2$. Using a spectral sequence, we link these two theories together in $\S 2.3$. Theorem A is proved in Theorem 2.11, Theorem 2.14 and Theorem 2.15. The filtration for the $\mathbb{Z}$-graded chain complex of the braid representing the composite knot is formulated in $\S 3.1$. Theorem $\mathrm{B}$ is proved in $\S 3.2$ as Theorem 3.9 and Proposition 3.10. $\S 4$ devotes to the proof of Theorem C (Theorem 4.3).

\section{The symplectic Floer homology of A BRAid}

2.1. The Floer homology of braids. We briefly recall our definition of the Floer homology of braids in this subsection. See 15 for more details.

For any knot $K=\bar{\beta}$ with $\beta \in B_{n}$ braid group, the space $\mathcal{R}\left(S^{2} \backslash K\right)^{[i]}$ can be identified with the space of $2 n$ matrices $X_{1}, \cdots, X_{n}, Y_{1}, \cdots, Y_{n}$ in $S U(2)$ satisfying

$$
\begin{gathered}
\operatorname{tr}\left(X_{i}\right)=\operatorname{tr}\left(Y_{i}\right)=0, \quad \text { for } i=1, \cdots, n, \\
X_{1} \cdot X_{2} \cdots X_{n}=Y_{1} \cdot Y_{2} \cdots Y_{n} .
\end{gathered}
$$

Let $\mathcal{R}^{*}\left(S^{2} \backslash K\right)^{[i]}$ be the subset of $\mathcal{R}\left(S^{2} \backslash K\right)^{[i]}$ consisting of irreducible representations. Note that $\pi_{1}\left(S^{2} \backslash K\right)$ is generated by $m_{x_{i}}, m_{y_{i}}(i=1,2, \cdots, n)$ with one relation $\prod_{i=1}^{n} m_{x_{i}}=$ $\prod_{i=1}^{n} m_{y_{i}}$. There is a unique reducible conjugacy class of representations $s_{K}: \pi_{1}\left(S^{3} \backslash K\right) \rightarrow$ $U(1)$ is the diagonal matrix

$$
s_{K}\left(\left[m_{x_{i}}\right]\right)=s_{K}\left(\left[m_{y_{i}}\right]\right)=\left(\begin{array}{cc}
i & 0 \\
0 & -i
\end{array}\right) .
$$

The space $\mathcal{R}^{*}\left(S^{2} \backslash K\right)^{[i]}$ is a monotone symplectic manifold of dimension $4 n-6$ by Lemma 2.3 in [15]. The symplectic manifold $(M, \omega)$ is called monotone if $\pi_{2}(M)=0$ or if there 
exists a nonnegative $\alpha \geq 0$ such that $I_{\omega}=\alpha I_{c_{1}}$ on $\pi_{2}(M)$, where $I_{\omega}(u)=\int_{S^{2}} u^{*}(\omega) \in \mathbb{R}$ and $I_{c_{1}}(u)=\int_{S^{2}} u^{*}\left(c_{1}\right) \in \mathbb{Z}$ for $u \in \pi_{2}(M)$. The braid $\beta$ induces a diffeomorphism $\phi_{\beta}$ : $\mathcal{R}^{*}\left(S^{2} \backslash K\right)^{[i]} \rightarrow \mathcal{R}^{*}\left(S^{2} \backslash K\right)^{[i]}$. The induced diffeomorphism $\phi_{\beta}$ is symplectic, and the fixed point set of $\phi_{\beta}$ is $\mathcal{R}^{*}\left(S^{3} \backslash K\right)^{[i]}$ (see Lemma 2.4 in [15]).

Let $H: \mathcal{R}^{*}\left(S^{2} \backslash K\right)^{[i]} \times \mathbb{R} \rightarrow \mathbb{R}$ be a $C^{\infty}$ time-dependent Hamiltonian function with $H(x, s)=H\left(\phi_{\beta}(x), s+1\right)$. Let $X_{s}$ be the corresponding vector field from $\omega\left(X_{s}, \cdot\right)=$ $d H_{s}(\cdot, s)$, and

$$
\frac{d \psi_{s}}{d s}=X_{s} \circ \psi_{s}, \quad \psi_{0}=i d
$$

Then we have $\psi_{s+1} \circ \phi_{\beta}^{H}=\phi_{\beta} \circ \psi_{s}$, where $\phi_{\beta}^{H}=\psi_{1}^{-1} \circ \phi_{\beta}$. Let $\Omega_{\phi_{\beta}}$ be the space of smooth contractible paths $\alpha$ in $\mathcal{R}^{*}\left(S^{2} \backslash K\right)^{[i]}$ such that $\alpha(s+1)=\phi_{\beta}(\alpha(s))$. The symplectic action $a_{H}: \Omega_{\phi_{\beta}} \rightarrow \mathbb{R} / \alpha 2 N \mathbb{Z}$ is given by

$$
d a_{H}(\gamma) \xi=\int_{0}^{1} \omega\left(\dot{\gamma}-X_{s}(\gamma), \xi\right) d s
$$

So the critical points of $a_{H}$ are the fixed points of $\phi_{\beta}^{H}$. For $x \in \operatorname{Fix}\left(\phi_{\beta}^{H}\right)$, define $\mu(x)=$ $\mu_{u}\left(x, s_{K}\right)(\bmod 2 N)$, where $\mu_{u}$ is the Maslov index and $N=N(K)$ is the first Chern number of the tangent bundle of $\mathcal{R}^{*}\left(S^{2} \backslash K\right)^{[i]}$. The symplectic Floer chain complex is defined by

$$
C_{j}=\left\{x \in \operatorname{Fix}\left(\phi_{\beta}\right) \cap \mathcal{R}^{*}\left(S^{2} \backslash K\right)^{[i]}: \mu(x)=j \in \mathbb{Z}_{2 N}\right\} .
$$

The following is the Proposition 4.1 and Theorem 4.2 in [15].

Theorem 2.1. For a knot $K=\bar{\beta}$, there is a well-defined $\mathbb{Z}_{2 N}$-graded symplectic Floer homology $\mathrm{HF}_{*}^{\text {sym }}\left(\phi_{\beta}^{H}\right)$. The symplectic Floer homology $\left\{H F_{j}^{\text {sym }}\left(\phi_{\beta}\right)\right\}_{j \in \mathbb{Z}_{2 N}}$ is a knot invariant and its Euler number is half of the signature of the knot $K$.

2.2. The $\mathbb{Z}$-graded symplectic Floer homology of braids. In this subsection, we extend our $\mathbb{Z}_{2 N}$-graded Floer homology to the $\mathbb{Z}$-graded symplectic Floer homology of braids provided $\alpha N \neq 0$. If $\alpha N=0$, then the symplectic Floer homology of braids defined in [15] is $\mathbb{Z}$-graded. Using a compatible filtration, we obtain a spectral sequence. The $E^{1}$ term of the spectral sequence is the $\mathbb{Z}$-graded Floer homology of a braid, and $E^{\infty}$ gives the $\mathbb{Z}_{2 N}$-graded Floer homology.

Lemma 2.2. The symplectic manifold $\mathcal{R}^{*}\left(S^{2} \backslash K\right)^{[i]}$ is path connected.

Proof: We prove this result by induction on the braid group $B_{n}$. For $n=2$ (i.e., the knot $K$ can be represented by $\left.K=\overline{\sigma_{1}^{\varepsilon(K)}}\right)$, we see that $\mathcal{R}^{*}\left(S^{2} \backslash K\right)^{[i]}$ is a pillow case in [15, 20]. So the manifold $\mathcal{R}^{*}\left(S^{2} \backslash K\right)^{[i]}$ is path connected. In particular, the space $\mathcal{R}^{*}\left(S^{2} \backslash K\right)^{[i]}$ is $K\left(\mathbb{Z}^{3}, 1\right)$ space. 
Suppose $n-1$ is true. For $\mathcal{R}^{*}\left(S^{2} \backslash K\right)^{[i]}=\left(H_{n} \backslash S_{n}\right) / S U(2)$ in Lin's notation [20], we have $\left(X_{1}, \cdots, X_{n}, Y_{1}, \cdots, Y_{n}\right)$ satisfies

$$
\begin{gathered}
\operatorname{tr}\left(X_{j}\right)=\operatorname{tr}\left(Y_{j}\right)=0, \quad j=1, \cdots, n, \\
X_{1} \cdots X_{n}=Y_{1} \cdots Y_{n} .
\end{gathered}
$$

Applying the conjugate operation on $X_{n}$ and $Y_{n}$, we may assume that

$$
X_{n}=\left(\begin{array}{cc}
i & 0 \\
0 & -i
\end{array}\right), \quad Y_{n}=\left(\begin{array}{cc}
i \cos \theta & \sin \theta \\
-\sin \theta & -i \cos \theta
\end{array}\right), \quad 0 \leq \theta \leq \pi
$$

If $\theta=0$ and $\pi$, then we get two copies of $\left(H_{n-1} \backslash S_{n-1}\right) / S U(2)$ which is path connected by the inductive hypothesis. For $0<\theta<\pi$, the equation (3) becomes

$$
X_{1} \cdots X_{n-1}\left(\begin{array}{cc}
\cos \theta & -i \sin \theta \\
-i \sin \theta & \cos \theta
\end{array}\right)=Y_{1} \cdots Y_{n-1} .
$$

Let $R_{\theta}$ be the representations in $\mathcal{R}^{*}\left(S^{2} \backslash K\right)^{[i]}$ satisfying the above equation. So the space $R_{\theta}$ is non-singular piece in $\mathcal{R}^{*}\left(S^{2} \backslash K\right)^{[i]}$. For $0<\theta, \theta^{\prime}<\pi$, the space $R_{\theta}$ is diffeomorphic to the space $R_{\theta^{\prime}}$. In particular, they are all diffeomorphic to $R_{\pi / 2}$. In this case, we see that $\mathcal{R}^{*}\left(S^{2} \backslash K\right)^{[i]}$ is a generalized pillow case:

$$
\mathcal{R}^{*}\left(S^{2} \backslash K\right)^{[i]}=\bigcup_{0 \leq \theta \leq \pi} R_{\theta} .
$$

It is clear that elements in $R_{\theta}$ can be path connected to elements in $R_{\theta^{\prime}}$. So the manifold $\mathcal{R}^{*}\left(S^{2} \backslash K\right)^{[i]}$ is path connected.

I owe this idea to X-S. Lin. For the path connected manifold $\mathcal{R}^{*}\left(S^{2} \backslash K\right)^{[i]}$ and $\alpha_{i} \in \Omega_{\phi_{\beta}}$ $(i=0,1)$, there is a path $\gamma$ in $\mathcal{R}^{*}\left(S^{2} \backslash K\right)^{[i]}$ connecting $\alpha_{0}(0)$ to $\alpha_{1}(0)$. The space $\Omega_{\phi_{\beta}}$ is path connected so that $\pi_{1}\left(\Omega_{\phi_{\beta}}, \alpha_{0}\right)$ is independent of a based point $\alpha_{0} \in \Omega_{\phi_{\beta}}$. Let $z_{0} \in \operatorname{Fix}\left(\phi_{\beta}\right) \subset \Omega_{\phi_{\beta}}$ be the base point.

Lemma 2.3. There exists a universal covering space $\Omega_{\phi_{\beta}}^{*}$ of $\Omega_{\phi_{\beta}}$.

Proof: The function space $\Omega_{\phi_{\beta}}=\operatorname{Map}\left(I, 0,1 ; \mathcal{R}^{*}\left(S^{2} \backslash K\right)^{[i]}, z_{0}\right)$ has the homotopy type of a CW complex and so an associated universal covering space. By Milnor's theorem 3.1 in [21], there is a universal covering space $\Omega_{\phi_{\beta}}^{*}$ of $\Omega_{\phi_{\beta}}$. Note that over this CW complex there is a universal covering space and so we can pull-back via the homotopy equivalence to the covering space over $\operatorname{Map}\left(I, 0,1 ; \mathcal{R}^{*}\left(S^{2} \backslash K\right)^{[i]}, z_{0}\right)$. As long as we work in the CW category this covering space is as good as the universal covering space.

Using the standard algebraic topology in [26], we can identify the transformation group of the universal covering space of $\Omega_{\phi_{\beta}}$.

Lemma 2.4. The transformation group of $\Omega_{\phi_{\beta}}^{*}$ is $\pi_{2}\left(\mathcal{R}^{*}\left(S^{2} \backslash K\right)^{[i]}, z_{0}\right)$. 
Proof: Let $u(t) \in \Omega_{\phi_{\beta}}$ be a map from $I$ to $\mathcal{R}^{*}\left(S^{2} \backslash K\right)^{[i]}$ with $u(t)(0)=u(t)(1)=z_{0}$ and $u(t)(s)$ is contractible path as a path in the variable $s$. Any loop $u(t) \in \Omega_{\phi_{\beta}}$ with $u(0)=u(1)=z_{0} \in \operatorname{Fix}\left(\phi_{\beta}\right)$ is a map

$$
u:(I \times I, I \times\{0,1\} \cup\{0,1\} \times I) \rightarrow\left(\mathcal{R}^{*}\left(S^{2} \backslash K\right)^{[i]}, z_{0}\right) .
$$

Note that the set of homotopy classes of maps in (4) is in one-to-one correspondence with $\pi_{2}\left(\mathcal{R}^{*}\left(S^{2} \backslash K\right)^{[i]}, z_{0}\right)$ (see [26]).

The transformation group $\pi_{2}\left(\mathcal{R}^{*}\left(S^{2} \backslash K\right)^{[i]}, z_{0}\right)$ is abelian. From the symplectic action map $a_{H}: \Omega_{\phi_{\beta}} \rightarrow \mathbb{R} / \alpha(2 N) \mathbb{Z}$, we pullback the universal covering space of $\mathbb{R} / \alpha(2 N) \mathbb{Z} \cong S^{1}$ over $\Omega_{\phi_{\beta}}$ :

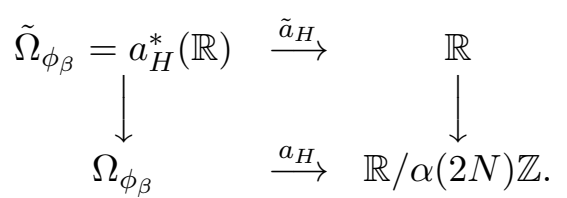

The pullback $a_{H}^{*}(\mathbb{R})=\tilde{\Omega}_{\phi_{\beta}} \rightarrow \Omega_{\phi}$ is an infinite cyclic subcovering space of the abelian universal covering space $\Omega_{\phi_{\beta}}^{*}$ with

$$
\pi_{1}\left(\tilde{\Omega}_{\phi_{\beta}}\right)=\mathbb{Z} \triangleleft \pi_{1}\left(\Omega_{\phi_{\beta}}^{*}\right)=\pi_{2}\left(\mathcal{R}^{*}\left(S^{2} \backslash K\right)^{[i]}, z_{0}\right) .
$$

Now the closed 1-form $d a_{H}(z)$ has a functional $\tilde{a}_{H}$ up to a constant such that $\tilde{a}_{H}: \tilde{\Omega}_{\phi_{\beta}} \rightarrow$ $\mathbb{R}$ is well-defined. By adding a constant, we assume $\tilde{a}_{H}\left(z_{0}\right)=0$. For a transformation element $g \in \pi_{1}\left(\tilde{\Omega}_{\phi_{\beta}}\right) \triangleleft \pi_{2}\left(\mathcal{R}^{*}\left(S^{2} \backslash K\right)^{[i]}\right)$, we obtain

$$
\tilde{a}_{H}(g(x))=\tilde{a}_{H}(x)+\operatorname{deg}(g) 2 \alpha N,
$$

where $\operatorname{deg}(g)$ is defined as $I_{\omega}(g)=\operatorname{deg}(g) \alpha(2 N)$. Let $\operatorname{Im} \tilde{a}_{H}\left(\right.$ Fix $\left.\phi_{\beta}\right)$ be the image of $\tilde{a}_{H}$ of $\widetilde{\operatorname{Fix} \phi_{\beta}}$; modulo $2 \alpha N \mathbb{Z}, \operatorname{Im} \tilde{a}_{H}\left(\widetilde{\operatorname{Fix} \phi_{\beta}}\right)$ is a finite set. Thus a set $\mathbb{R}_{\phi_{\beta}}=\mathbb{R} \backslash \operatorname{Im} \tilde{a}_{H}\left(\widetilde{\operatorname{Fix} \phi_{\beta}}\right)$ consists of the regular values of the symplectic action $\tilde{a}_{H}$ on $\tilde{\Omega}_{\phi_{\beta}}$. We construct a $\mathbb{Z}$-graded symplectic Floer cohomology for every $r \in \mathbb{R}_{\phi_{\beta}}$.

Given $x \in \operatorname{Fix} \phi_{\beta} \subset \Omega_{\phi_{\beta}}$, let $x^{(r)} \in \widetilde{\operatorname{Fix} \phi_{\beta}} \subset \tilde{\Omega}_{\phi_{\beta}}$ be the unique lift of $x$ such that $\tilde{a}_{H}\left(x^{(r)}\right) \in(r, r+2 \alpha N)$. Note that if $\alpha N=0$ then one already has an $\mathbb{Z}$-graded symplectic Floer homology as in [15], so we work on the case $\alpha N \neq 0$. To cover the case $\alpha N \neq 0$, we may choose $r<\min \left\{a_{H}\left(\operatorname{Fix} \phi_{\beta}\right),(\bmod 2 \alpha N)\right\}$ in the interval $(0,2 \alpha N)$. Then there exists a unique element $x$ such that $\tilde{a}_{H}\left(x^{(r)}\right) \in(r, r+2 \alpha N)$. Let $\mu^{(r)}(x)=\mu\left(x^{(r)}, s_{K}\right) \in \mathbb{Z}$ (the unique reducible conjugacy class $s_{K}$ of representations in $\left.\mathcal{R}\left(S^{3} \backslash K\right)^{[i]}\right)$ and define the $\mathbb{Z}$-graded symplectic Floer chain group

$$
C_{n}^{(r)}\left(\phi_{\beta}\right)=\mathbb{Z}\left\{x \in \operatorname{Fix} \phi_{\beta} \quad \mid \quad \mu^{(r)}(x)=n \in \mathbb{Z}\right\},
$$

as the free $\mathbb{Z}$ module generated by $x \in \operatorname{Fix} \phi_{\beta}$ with the lift $x^{(r)}$ and $\mu\left(x^{(r)}, s_{K}\right)=n$.

If $\overline{z_{0}}\left(\bar{s}_{K}\right)$ is another choice of a based point $z_{0}$ (respectively $s_{K}$ for the Maslov index) and $g\left(z_{0}\right)=\overline{z_{0}}\left(g\left(s_{K}\right)=\bar{s}_{K}\right)$ for some covering transformation $g \in \pi_{1}\left(\tilde{\Omega}_{\phi_{\beta}}\right)$, then the 
corresponding choice of lift $\bar{x}^{(r)}$ of $x$ is just $g\left(x^{(r)}\right)$ by the uniqueness. Note that the integral Maslov index $\mu_{u}^{(r)}(x)$ is independent of the choice of the based point $z_{0}\left(s_{K}\right)$ used in the definition of $a_{H}$ by (5). We may choose $z_{0}=s_{K}$ for simplicity on both lifts of the symplectic action and the Maslov index (see [9). The following lemma shows that the lift of the functional $a_{H}$ is compatible with a universal lift of $\mathbb{R} / 2 \alpha N Z\left(\cong S^{1}\right)$ via the monotonicity of the symplectic manifold $\mathcal{R}^{*}\left(S^{2} \backslash K\right)^{[i]}$.

Lemma 2.5. The lift of the symplectic action over $\tilde{\Omega}_{\phi_{\beta}}$ is compatible with the one of the Maslov index: for $g \in \pi_{2}\left(\mathcal{R}^{*}\left(S^{2} \backslash K\right)^{[i]}\right)=\pi_{1}\left(\tilde{\Omega}_{\phi_{\beta}}\right)$ with $\operatorname{deg}(g)=n$ in the sense of (包),

$$
\tilde{a}_{H}\left(g\left(s_{K}\right)\right)=n \alpha(2 N) \quad \text { if and only if } \quad \mu^{(r)}\left(g\left(s_{K}\right), s_{K}\right)=n(2 N) .
$$

Proof: The result follows from the definition of $\tilde{\Omega}_{\phi_{\beta}}$ and the monotonicity of $\left(\mathcal{R}^{*}\left(S^{2} \backslash\right.\right.$ $\left.K)^{[i]}, \omega\right)$ (see Lemma 2.3 in [15]). So the Maslov index also provides an integral lifting of element $x \in \operatorname{Fix}\left(\phi_{\beta}\right)$.

Definition 2.6. The integral Floer boundary map $\partial^{(r)}: C_{n+1}^{(r)}\left(\phi_{\beta}\right) \rightarrow C_{n}^{(r)}\left(\phi_{\beta}\right)$ is defined by

$$
\partial^{(r)} x=\sum_{y \in C_{n}^{(r)}\left(\phi_{\beta}\right)} \# \hat{\mathcal{M}}(x, y) \cdot y,
$$

where $\mathcal{M}(x, y)$ denote the union of the components of 1-dimensional moduli space of $J$ holomorphic curves and $\hat{\mathcal{M}}(x, y)=\mathcal{M}(x, y) / \mathbb{R}$ is a zero dimensional moduli space modulo the time translation. The number $\# \hat{\mathcal{M}}(x, y)$ counts the points with sign in [8, 15].

Note that the boundary map $\partial^{(r)}$ only counts part of the boundary map in Proposition 4.1 of 15. We are going to show that $\partial^{(r)} \circ \partial^{(r)}=0$. The corresponding homology groups are the $\mathbb{Z}$-graded symplectic Floer homology $I_{*}^{(r)}\left(\phi_{\beta}\right), * \in \mathbb{Z}$. See [14, 24] for the following.

Proposition 2.7. Suppose that elements in Fix $\left(\phi_{\beta}\right)$ are nondegenerate. (i) If $u \in \mathcal{P}(x, y)$ for $x, y \in$ Fix $\phi_{\beta}$ and $\tilde{u}$ is any lift of $u$, then $\mu_{\tilde{u}}=\mu^{(r)}(y)-\mu^{(r)}(x)$.

(ii) Then there is a dense subset $\mathcal{J}_{*}(\phi) \subset \mathcal{J}_{\text {reg }}\left(\phi_{\beta}\right)$ of $\mathcal{J}$ such that (1) the zero dimensional component of $\hat{\mathcal{M}}(x, y)$ is compact and (2) the one dimensional component of $\hat{\mathcal{M}}\left(x^{\prime}, y^{\prime}\right)$ is compact up to the splitting of two isolated trajectories for $J \in \mathcal{J}_{*}\left(\phi_{\beta}\right)$.

Proposition 2.7 plays the key role in showing that $\partial \circ \partial=0$ (see [15] $\S 4$ ). We follow the same argument in [9, 15] to show that $\partial^{(r)} \circ \partial^{(r)}=0$.

Lemma 2.8. Under the same hypothesis in Proposition 2.X, $\partial^{(r)} \circ \partial^{(r)}=0$. is

Proof: If $x \in C_{n+1}^{(r)}\left(\phi_{\beta}\right)$, then by definition the coefficient of $z \in C_{n-1}^{(r)}\left(\phi_{\beta}\right)$ in $\partial^{(r)} \circ \partial^{(r)}(x)$

$$
\sum_{y \in C_{n}^{(r)}\left(\phi_{\beta}\right)} \# \hat{\mathcal{M}}(x, y) \cdot \# \hat{\mathcal{M}}(y, z) .
$$


By Proposition 2.7, the boundary of the 1-dimensional manifold $\hat{\mathcal{M}}(x, z)=\mathcal{M}(x, z) / \mathbb{R}$ corresponds to two isolated trajectories $\mathcal{M}(x, y) \times \mathcal{M}(y, z)$. Each term $\# \hat{\mathcal{M}}(x, y) \cdot \# \hat{\mathcal{M}}(y, z)$ is the number of the 2-cusp trajectory of $\hat{\mathcal{M}}(x, z)$ with $y \in C_{n}^{(r)}\left(\phi_{\beta}\right)$. For any such $y$ there are $J$-holomorphic curves $u \in \mathcal{M}(x, y)$ and $v \in \mathcal{M}(y, z)$. The other end of the corresponding component of the 1-manifold $\hat{\mathcal{M}}(x, z)$ corresponds to the splitting $\mathcal{M}\left(x, y^{\prime}\right) \times \mathcal{M}\left(y^{\prime}, z\right)$ with $u^{\prime} \in \mathcal{M}\left(x, y^{\prime}\right)$ and $v^{\prime} \in \mathcal{M}\left(y^{\prime}, z\right)$. It is impossible for $y^{\prime}$ to be the reducible $s$ because the $U(1)$ symmetry group would add one more parameter to the moduli space. Then $\hat{\mathcal{M}}(x, z)$ has an one parameter family of paths from $x$ to $z$ with ends $u \# v$ and $u^{\prime} \# v^{\prime}$ for appropriate grafting. If we lift $u$ to $\tilde{u} \in \tilde{\mathcal{M}}\left(x^{(r)}, \tilde{y}\right)$, then

$$
1=\mu_{\tilde{u}}=\mu^{(r)}(x)-\mu^{(r)}(\tilde{y})=(n+1)-\mu^{(r)}(\tilde{y}) .
$$

So $\mu^{(r)}(\tilde{y})=n$; and $\tilde{y}=y^{(r)}$ is the preferred lift, thus we have $\tilde{u} \in \tilde{\mathcal{M}}\left(x^{(r)}, y^{(r)}\right)$. Similarly $\tilde{v} \in \tilde{\mathcal{M}}\left(y^{(r)}, z^{(r)}\right)$. Since $u^{\prime} \# v^{\prime}$ is homotopic to $u \# v$ rel $\left(x^{(r)}, z^{(r)}\right)$, the lift $\tilde{u}^{\prime} \# \tilde{v}^{\prime}$ is also a path with ends $\left(x^{(r)}, z^{(r)}\right)$. The symplectic action $a_{H}$ is non-decreasing along the gradient trajectory $\tilde{u}^{\prime}$ with $\tilde{u}^{\prime}(-\infty)=x^{(r)}$ and $\tilde{u}^{\prime}(+\infty)=\tilde{y}^{\prime}$, we have

$$
r<a\left(x^{(r)}\right) \leq a\left(\tilde{y}^{\prime}\right) \leq a\left(z^{(r)}\right)<r+\alpha 2 N
$$

By the uniqueness, $\tilde{y}^{\prime}=\left(y^{\prime}\right)^{(r)}$ and using (8) for $u^{\prime}$, we have $\mu^{(r)}\left(\left(y^{\prime}\right)^{(r)}\right)=\mu^{(r)}\left(x^{(r)}\right)+1=n$; so $\left(y^{\prime}\right)^{(r)} \in C_{n}^{(r)}\left(\phi_{\beta}\right)$. Thus the algebraic number of two-trajectories connecting $x^{(r)}$ and $z^{(r)}$ with index 2 is zero by the orientation discussed in 8 .

Now $\left(C_{n}^{(r)}\left(\phi_{\beta}\right), \partial_{n}^{(r)}\right)_{n \in \mathbb{Z}}$ is indeed a $\mathbb{Z}$-graded symplectic Floer chain complex of the braid $\beta \in B_{n}$. We call its homology to be a $\mathbb{Z}$-graded symplectic Floer homology, denoted by

$$
I_{*}^{(r)}\left(\phi_{\beta}\right)=H^{*}\left(C_{*}^{(r)}\left(\phi_{\beta}\right), \partial^{(r)}\right), \quad * \in \mathbb{Z} .
$$

From the construction we have that

$$
\begin{aligned}
& \text { (1) if }[r, s] \subset \mathbb{R}_{\phi} \text {, then } I_{*}^{(r)}\left(\phi_{\beta}\right)=I_{*}^{(s)}\left(\phi_{\beta}\right) ; \\
& \text { (2) } I_{*+(2 N)}^{(r)}\left(\phi_{\beta}\right)=I_{*}^{(r+\alpha(2 N))}\left(\phi_{\beta}\right),
\end{aligned}
$$

where $\alpha(2 N)(>0)$ is the minimal number in $\left.\operatorname{Im} I_{\omega}\right|_{\pi_{2}\left(\mathcal{R}^{*}\left(S^{2} \backslash K\right)^{[i]}\right)}$. The relation between $I_{*}^{(r)}\left(\phi_{\beta}\right)$ and $H F_{*}^{\mathrm{sym}}\left(\phi_{\beta}\right)$ will be discussed in $\S 2.3$. In the following, we notice that the $\mathbb{Z}$-graded symplectic Floer homology $I_{*}^{(r)}\left(\phi_{\beta}\right)$ is not a knot invariant in general.

Let $\left\{\left(J^{\lambda}, H^{\lambda}\right\}_{\lambda \in \mathbb{R}}\right.$ be an 1-parameter family that interpolates from $\left(J^{0}, H^{0}\right)$ to $\left(J^{1}, H^{1}\right)$ and is constant in $\lambda$ outside $[0,1]$. Define the perturbed $J$-holomorphic curve equations

$$
\bar{\partial}_{J \lambda} u_{\lambda}(s, t)=\frac{\partial u_{\lambda}}{\partial t}+J_{s}^{\lambda}\left(\frac{\partial u_{\lambda}}{\partial s}-X_{s}^{\lambda}\left(u_{\lambda}\right)\right)=0,
$$

with the moving conditions $u_{\lambda}(s+1, t)=\phi_{1}^{\lambda}\left(u_{\lambda}(s, t)\right)$ and the asymptotic values

$$
\lim _{t \rightarrow-\infty} u_{\lambda}(s, t)=x_{0} \in \operatorname{Fix}\left(\phi_{\beta, H^{0}}\right), \quad \lim _{t \rightarrow+\infty} u_{\lambda}(s, t)=x_{1} \in \operatorname{Fix}\left(\phi_{\beta, H^{1}}\right) .
$$


This directly generalizes the $J$-holomorphic curve equation in the case of $\left(J^{0}, \phi_{\beta, H^{0}}\right)$ and $\left(J^{1}, \phi_{\beta, H^{1}}\right)$ corresponding to $\left\{\left(J^{\lambda}, H^{\lambda}\right\}_{\lambda \in \mathbb{R}}\right.$. We define

$$
C_{\phi_{\beta}}^{(r)}=\min \left\{\tilde{a}_{H}\left(x^{(r)}\right)-r, 2 \alpha N+r-\tilde{a}_{H}\left(x^{(r)}\right) \mid x \in \operatorname{Fix}\left(\phi_{\beta}\right)\right\},
$$

where $H$ is the Hamiltonian function which makes all Fix $\left(\phi_{\beta}\right)$ nondegenerate and isolated. So the number $C_{\phi_{\beta}}^{(r)}$ is not dependent on $\left(J^{\lambda}, H^{\lambda}\right)$.

For each $x \in \operatorname{Fix}\left(\phi_{\beta}\right)$, there is an open neighborhood $U_{x}$ in $\Omega_{\phi_{\beta}}$ such that (1) $U_{x}$ is evenly covered in $\tilde{\Omega}_{\phi_{\beta}}$, (2) for each $z \in U_{x},\left|a_{H^{\lambda}}(z)-a_{H^{\lambda}}(x)\right|<C_{\phi_{\beta}}^{(r)} / 8$. There are finite subcover $\left\{U_{x_{1}}, \cdots, U_{x_{k}}\right\}$ of Fix $\left(\phi_{\beta}\right)$, and by Gromov's compactness theorem [6, 14, 24] we have $\varepsilon_{1}>0$ such that if $\left\|D a_{H^{\lambda}}(z)\right\|_{L_{1}^{3}}<\varepsilon_{1}$ then $z \in \bigcup_{i=1}^{k} U_{x_{i}}$. Let $\varepsilon=\min \left\{\varepsilon_{1}, C_{\phi_{\beta}}^{(r)} / 8\right\}$. We set a deformation $\left\{J^{\lambda}, H^{\lambda}\right\}$ satisfying the usual perturbation requirements in [6, 14], and also satisfying

$$
(i)\left|H_{t}^{\lambda}(z)\right|<\varepsilon / 2, \quad(i i)\left\|\nabla_{J} H_{t}^{\lambda}(z)\right\|_{L_{1}^{3}}<\varepsilon / 2,
$$

for all $z \in \Omega_{\phi_{\beta}}$. These deformation conditions can be achieved by the density statement in 6, 14, 24.

Let $\mathcal{P}_{1, \varepsilon / 2}$ be the set of $\left\{J^{\lambda}, H^{\lambda}\right\}$ which satisfies these extra conditions (14). The moduli space $\mathcal{M}_{\lambda}(x, y)$ of (12) and (13) has the same analytic properties as the moduli space $\mathcal{M}(x, y)$ except for the translational invariance.

Definition 2.9. For each $n$ and $\left\{J^{\lambda}, H^{\lambda}\right\} \in \mathcal{P}_{1, \varepsilon / 2}$, let

$$
\phi_{01}^{n}\left(x_{0}\right)=\sum_{x_{1} \in C_{n}^{(r)}\left(\phi_{\beta} ; J^{1}, H^{1}\right)} \# \mathcal{M}_{\lambda}^{0}\left(x_{0}, x_{1}\right) \cdot x_{1},
$$

be a homomorphism $\phi_{01}^{n}: C_{n}^{(r)}\left(\phi_{\beta} ; J^{0}, H^{0}\right) \rightarrow C_{n}^{(r)}\left(\phi_{\beta} ; J^{1}, H^{1}\right)$, where $\# \mathcal{M}_{\lambda}^{0}\left(x_{0}, x_{1}\right)$ is the algebraic number of the moduli space $\mathcal{M}_{\lambda}^{0}\left(x_{0}, x_{1}\right)$ with compatible orientation given by [8].

Proposition 2.10. For any continuation $\left(J^{\lambda}, H^{\lambda}\right) \in \mathcal{P}_{1, \varepsilon / 2}$ which is regular at the ends,

(i) the homomorphism $\left\{\phi_{01}^{*}\right\}_{* \in Z}$ is a cochain map, i.e.,

$$
\partial_{n, 1}^{(r)} \circ \phi_{01}^{n}=\phi_{01}^{n} \circ \partial_{n, 0}^{(r)}
$$

for all $n \in Z$;

(ii) for another regular pair $\left(J^{\lambda_{1}}, H^{\lambda_{1}}\right)$ connecting $\left(J^{1}, H^{1}\right)$ to $\left(J^{2}, H^{2}\right)$ in $\mathcal{P}_{1, \varepsilon / 2}$,

$$
\phi_{12}^{n} \circ \phi_{01}^{n}=\phi_{02}^{n}, \quad \phi_{00}^{n}=i d .
$$

Proof: The proof follows from the same argument in the theorem 4 of [15] and the method of the proof of Lemma 2.8. See [6, 9].

By Proposition 2.10, the $\mathbb{Z}$-graded symplectic Floer homology $\left\{I_{n}^{(r)}\left(\phi_{\beta}\right)\right\}_{n \in \mathbb{Z}}$ is invariant under the continuation of $\left\{J^{\lambda}, H^{\lambda}\right\} \in \mathcal{P}_{1, \varepsilon / 2}$. 
Proposition 2.11. The $\mathbb{Z}$-graded symplectic Floer homology $\left\{I_{n}^{(r)}\left(\phi_{\beta}\right)\right\}_{n \in \mathbb{Z}}$ is invariant under the Markov move of type I and its inverse.

Proof: Suppose that we have a Markov move of type I: change $\beta$ to $\xi^{-1} \beta \xi$ for some $\xi \in B_{n}$. The element $\xi$ induces a diffeomorphism $\xi: Q_{n} \rightarrow Q_{n}$, and a symplectic diffeomorphism $\xi \times \xi: Q_{n} \times Q_{n} \rightarrow Q_{n} \times Q_{n}$ which commutes with the $S U(2)$ action (see 15 §4). Let $f_{\xi}: \mathcal{R}^{*}\left(S^{2} \backslash K\right)^{[i]} \rightarrow \mathcal{R}^{*}\left(S^{2} \backslash K\right)^{[i]}$ be the symplectic diffeomorphism induced from $\xi \times \xi$. We have

$$
\phi_{\beta}=f_{\xi} \circ \phi_{\xi^{-1} \beta \xi} \circ f_{\xi}^{-1}
$$

by changing variables via $f_{\xi}$. So $\tilde{\phi}_{\beta}=f_{\xi} \circ \tilde{\phi}_{\xi^{-1} \beta \xi} \circ f_{\xi}^{-1}$ for Hamiltonian perturbations. Thus $f_{\xi}$ induces a natural identification between $C_{n}^{(r)}\left(\phi_{\beta}\right)$ and $C_{n}^{(r)}\left(\phi_{\xi^{-1} \beta \xi}\right)$ since $\operatorname{Fix}\left(\phi_{\xi^{-1} \beta \xi}\right)$ can be identified with $\operatorname{Fix}\left(\phi_{\beta}\right)$ under $f_{\xi}$. Using the argument in Lemma 2.8 and [6], the induced map $f_{\xi}$ is a chain map, i.e., it commutes with the $\mathbb{Z}$-graded boundary maps. There is an inverse chain map $f_{\xi^{-1}}$ of $f_{\xi}$, and an isomorphism

$$
I_{n}^{(r)}\left(\phi_{\beta}\right) \cong I_{n}^{(r)}\left(\phi_{\xi^{-1} \beta \xi}\right) .
$$

It is clear that the argument goes through for the inverse operation of a Markov move of type I.

For the Markov move of type II (change $\beta$ to $\sigma_{n} \beta \in B_{n+1}$ ), there is an imbedding $g: Q_{n} \times Q_{n} \rightarrow Q_{n+1} \times Q_{n+1}$ which induces an imbedding

$$
\hat{g}: \hat{H}_{n}\left(=\mathcal{R}^{*}\left(S^{2} \backslash \bar{\beta}\right)^{[i]}\right) \rightarrow \hat{H}_{n+1}\left(=\mathcal{R}^{*}\left(S^{2} \backslash \overline{\sigma_{n} \beta}\right)^{[i]}\right) .
$$

See 15] for more details. The image $\hat{g}\left(\phi_{\beta}\right)$ is invariant under the operation of $\sigma_{n}$. The symplectic diffeomorphism $\phi_{\sigma_{n} \beta}$ can be connected with $\hat{g}\left(\phi_{\beta}\right)$ through a Hamiltonian isotopy $\psi_{t}$ in 15. Such a Hamiltonian isotopy $\psi_{t}$ is constructed by a rotation about an axis. The Hamiltonian flow induced by $\psi_{t}$ connects two general points in $S^{2}$. The Hamiltonian function $H$ is $x_{3}$ or $g x_{3}$ for $g \in S O(3)$. The function $H$ does not satisfy (14) for small $\varepsilon$ $(\nabla H=(0,0,1))$.

In [25], Smale proves that the rotation group $S O(3)$ is a strong deformation retract of the orientation preserving $C^{\infty}$-diffeomorphisms of $S^{2}$ with $C^{r}$ topology. Since the 2 -sphere is simply connected, so every symplectic diffeomorphism of identity component is Hamiltonian. Thus any Hamiltonian isotopy connecting $\hat{g}\left(\phi_{\beta}\right)$ with $\phi_{\sigma_{n} \beta}$ can be obtained by the rotation about certain axis. I.e., the Hamiltonian function is not a small perturbation for the Markov move of type II and its inverse. So the continuation $\left(J^{\lambda}, H^{\lambda}\right)$ realizing the Markov move of type II is not in $\mathcal{P}_{1, \varepsilon / 2}$ in general. It is interesting to investigate this large perturbation via the method developed in [1]. The homomorphism $\phi_{01}^{n}$ in Definition 2.9 is not welldefined. So the $\mathbb{Z}$-graded symplectic Floer homology of braids is not invariant under the Markov move of type II and its inverse. Although the $\mathbb{Z}$-graded symplectic Floer homology of braids is not a knot invariant, we formulate a spectral sequence which converges to a 
knot invariant in the next subsection. The $\mathbb{Z}$-graded symplectic Floer homology of a braid $\beta \in B_{n}$ is a stepping stone to compute the $\mathbb{Z}_{2 N}$-graded symplectic Floer homology of the knot $K=\bar{\beta}$.

2.3. The spectral sequence for the symplectic Floer homology. In this subsection we are going to show that the $\mathbb{Z}$-graded symplectic Floer homology $I_{*}^{(r)}\left(\phi_{\beta}\right)$ for $r \in \mathbb{R}_{\phi_{\beta}}$ and $* \in \mathbb{Z}$ determines the $\mathbb{Z}_{2 N}$-graded symplectic Floer homology $H F_{\text {sym }}^{*}\left(\phi_{\beta}\right)\left(* \in \mathbb{Z}_{2 N}\right)$. The way to link them together is to filter the $\mathbb{Z}$-graded symplectic Floer chain complex. The filtration, by a standard method in algebraic topology, formulates a spectral sequence which converges to the $\mathbb{Z}_{2 N}$-graded symplectic Floer homology $H F_{*}^{\text {sym }}\left(\phi_{\beta}\right)$ of the knot $K=\bar{\beta}$.

Definition 2.12. For $r \in \mathbb{R}_{\phi}, j \in \mathbb{Z}_{2 N}$ and $n \equiv j(\bmod (2 N))$, define the free $\mathbb{Z}$ modules

$$
F_{n}^{(r)} C_{j}\left(\phi_{\beta}\right)=\sum_{k \geq 0} C_{n+(2 N) k}^{(r)}\left(\phi_{\beta}\right)
$$

which gives a natural decreasing filtration on $C_{*}\left(\phi_{\beta}\right)\left(* \in \mathbb{Z}_{2 N}\right)$.

There is a finite length decreasing filtration of $C_{j}\left(\phi_{\beta}\right)\left(j \in \mathbb{Z}_{2 N}\right)$ :

$$
\begin{gathered}
\cdots F_{n+(2 N)}^{(r)} C_{j}\left(\phi_{\beta}\right) \subset F_{n}^{(r)} C_{j}\left(\phi_{\beta}\right) \subset F_{n-(2 N)}^{(r)} C_{j}\left(\phi_{\beta}\right) \cdots \subset C_{j}\left(\phi_{\beta}\right) . \\
C_{j}\left(\phi_{\beta}\right)=\bigcup_{n \equiv j} F_{n}^{(r)} C_{j}\left(\phi_{\beta}\right) .
\end{gathered}
$$

Note that the symplectic action is non-decreasing along the gradient trajectories, it follows that the boundary map $\partial: F_{n}^{(r)} C_{j}\left(\phi_{\beta}\right) \rightarrow F_{n-1}^{(r)} C_{j-1}\left(\phi_{\beta}\right)$ preserves the filtration in

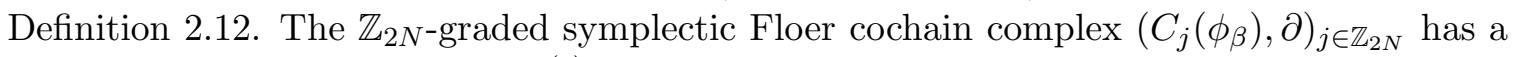
decreasing bounded filtration $\left(F_{n}^{(r)} C_{*}\left(\phi_{\beta}\right), \partial\right)$,

$$
\begin{array}{ccccc} 
& \downarrow & \downarrow & & \downarrow \\
\cdots & F_{n+2 N}^{(r)} C_{j}\left(\phi_{\beta}\right) & \subset F_{n}^{(r)} C_{j}\left(\phi_{\beta}\right) & \cdots & \subset C_{j}\left(\phi_{\beta}\right) \\
& \downarrow \partial^{(r)} & \downarrow \partial^{(r)} & & \downarrow \partial \\
\cdots & F_{n+(2 N)-1}^{(r)} C_{j-1}\left(\phi_{\beta}\right) & \subset F_{n-1}^{(r)} C_{j-1}\left(\phi_{\beta}\right) & \cdots & \subset C_{j-1}(\phi ; P, J) \\
\downarrow & \downarrow & \downarrow & & \downarrow
\end{array} .
$$

Lemma 2.13. (1) The homology of the vertical chain subcomplex $F_{n}^{(r)} C_{*}\left(\phi_{\beta}\right)$ in the filtration (17) is $F_{n}^{(r)} I_{j}^{(r)}\left(\phi_{\beta}\right)$.

(2) There is a natural bounded filtration for $\left\{I_{*}^{(r)}\left(\phi_{\beta}\right)\right\}_{* \in Z}$ the integral-graded symplectic Floer homology,

$$
\cdots F_{n+(2 N)}^{(r)} H F_{j}\left(\phi_{\beta}\right) \subset F_{n}^{(r)} H F_{j}\left(\phi_{\beta}\right) \subset F_{n-(2 N)}^{(r)} H F_{j}\left(\phi_{\beta}\right) \cdots \subset I_{j}^{(r)}\left(\phi_{\beta}\right),
$$

where $F_{n}^{(r)} H F_{j}\left(\phi_{\beta}\right)=\operatorname{Im}\left(F_{n}^{(r)} I_{j}^{(r)}\left(\phi_{\beta}\right) \rightarrow I_{j}^{(r)}\left(\phi_{\beta}\right)\right)$. 
Proof: The results follows from Definition 2.12 and standard results in 26] Chapter 9 and [9].

Theorem 2.14. (i) There is a spectral sequence $\left(E_{n, j}^{k}\left(\phi_{\beta}\right), d^{k}\right)$ with

$$
E_{n, j}^{1}\left(\phi_{\beta}\right) \cong I_{n}^{(r)}\left(\phi_{\beta}\right), \quad n \equiv j \quad(\bmod (2 N))
$$

and the higher differential $d^{k}: E_{n, j}^{k}\left(\phi_{\beta}\right) \rightarrow E_{n+(2 N) k-1, j-1}^{k}\left(\phi_{\beta}\right)$, and

$$
E_{n, j}^{\infty}\left(\phi_{\beta}\right) \cong F_{n}^{(r)} H F_{j}^{s y m}\left(\phi_{\beta}\right) / F_{n+(2 N)}^{(r)} H F_{j}^{s y m}\left(\phi_{\beta}\right) .
$$

(ii) The spectral sequence $\left(E_{n, j}^{k}\left(\phi_{\beta}\right), d^{k}\right)$ converges to $H F_{*}^{\text {sym }}\left(\phi_{\beta}\right)$ (the $\mathbb{Z}_{2 N}$-graded symplectic Floer homology) of the knot $K=\bar{\beta}$.

Proof: (i) Note that

$$
F_{n}^{(r)} C_{j}\left(\phi_{\beta}\right) / F_{n+(2 N)}^{(r)} C_{j}\left(\phi_{\beta}\right)=C_{n}^{(r)}\left(\phi_{\beta}\right) .
$$

It is standard from [26] that there exists a spectral sequence $\left(E_{n, j}^{k}, d^{k}\right)$ with $E^{1}$ term given by the homology of $F_{n}^{(r)} C_{j}\left(\phi_{\beta}\right) / F_{n+(2 N)}^{(r)} C_{j}\left(\phi_{\beta}\right)$, so we have $E_{n, j}^{1}\left(\phi_{\beta}\right) \cong I_{n}^{(r)}\left(\phi_{\beta}\right)$ and $E_{n, j}^{\infty}\left(\phi_{\beta}\right)$ is isomorphic to the bigraded $Z$-module associated to the filtration $F^{(r)}$ of the $\mathbb{Z}$-graded symplectic Floer homology $I_{n}^{(r)}\left(\phi_{\beta}\right)$.

(ii) Since $\operatorname{Fix} \phi_{\beta}$ is a finite set and non-degenerate, so the filtration $F$ is bounded and complete from (15) and (16). Thus the induced spectral sequence converges to the $\mathbb{Z}_{2 N^{-}}$ graded symplectic Floer homology. Note that the grading is unusual (jumping by $2 N$ in each step), we list the terms for $Z_{*, *}^{k}\left(\phi_{\beta}\right)$ and $E_{*, *}^{k}\left(\phi_{\beta}\right)$.

$$
\begin{aligned}
& Z_{n, j}^{k}\left(\phi_{\beta}\right)=\left\{x \in F_{n}^{(r)} C_{j}\left(\phi_{\beta}\right) \mid \partial x \in F_{n-1+(2 N) k}^{(r)} C_{j-1}\left(\phi_{\beta}\right)\right\}, \\
& Z_{n, j}^{\infty}\left(\phi_{\beta}\right)=\left\{x \in F_{n}^{(r)} C_{j}\left(\phi_{\beta}\right) \mid \partial x=0\right\}, \\
& E_{n, j}^{k}\left(\phi_{\beta}\right)=Z_{n, j}^{k}\left(\phi_{\beta}\right) /\left\{Z_{n+(2 N), j}^{k+1}\left(\phi_{\beta}\right)+\partial Z_{n+(k-1)(2 N)+1, j+1}^{k-1}\left(\phi_{\beta}\right)\right\}, \\
& E_{n, j}^{\infty}\left(\phi_{\beta}\right)=Z_{n, j}^{\infty}\left(\phi_{\beta}\right) /\left\{Z_{n+(2 N), j}^{\infty}\left(\phi_{\beta}\right)+\partial Z_{n+(k-1)(2 N)+1, j+1}^{\infty}\left(\phi_{\beta}\right)\right\} .
\end{aligned}
$$

Thus the Floer boundary map $\partial$ induces the higher differential

$$
d^{k}: E_{n, j}^{k}\left(\phi_{\beta}\right) \rightarrow E_{n+(2 N) k-1, j-1}^{k}\left(\phi_{\beta}\right) .
$$

Theorem 2.15. (1) For any continuation $\left(J^{\lambda}, H^{\lambda}\right) \in \mathcal{P}_{1, \varepsilon / 2}$ which is regular at ends, there exists an isomorphism

$$
E_{n, j}^{1}\left(\phi_{\beta} ; J^{0}, H^{0}\right) \cong E_{n, j}^{1}\left(\phi_{\beta} ; J^{1}, H^{1}\right) .
$$

(2) For each $k \geq 1, E_{n, j}^{k}\left(\phi_{\beta}\right)$ are the invariants under the continuation $\left(J^{\lambda}, H^{\lambda}\right) \in \mathcal{P}_{1, \varepsilon / 2}$. 
Proof: By Theorem 2.14, we have an isomorphism $E_{n, j}^{1}\left(\phi_{\beta} ; J^{0}, H^{0}\right) \cong I_{n}^{(r)}\left(\phi_{\beta} ; J^{0}, H^{0}\right)$, so there exists an isomorphism by Proposition 2.10: $I_{n}^{(r)}\left(\phi_{\beta} ; J^{0}, H^{0}\right) \rightarrow I_{n}^{(r)}\left(\phi_{\beta} ; J^{1}, H^{1}\right)$ which respects the filtration and induces an isomorphism on the $E^{1}$ term. Since $E_{n, j}^{1}\left(\phi_{\beta}\right)$ is an invariant under the continuation $\left(J^{\lambda}, H^{\lambda}\right) \in \mathcal{P}_{1, \varepsilon / 2}$ by Proposition 2.10 and Theorem 2.14, so (2) follows from Theorem 1 in [26] page 468.

If $\alpha N=0$, we already have the $\mathbb{Z}$-graded symplectic Floer homology $H F_{n}^{\text {sym }}\left(\phi_{\beta}\right)$ for $K=$ $\bar{\beta}$. All these new spectral sequences should contain more information on $\left(\mathcal{R}^{*}\left(S^{2} \backslash K\right)^{[i]} ; \phi_{\beta}\right)$, they are also finer than $H F_{*}^{\mathrm{sym}}\left(\phi_{\beta}\right), * \in \mathbb{Z}_{2 N}$ the symplectic homology defined in [15]. If $\alpha N \neq 0$, we only have that $\left\{E_{n, j}^{k}\left(\phi_{\beta}\right)\right\}$ is invariant under the Markov move of type I and its inverse by Proposition 2.11 and Theorem 1 in [26] page 468. In general $\left\{E_{n, j}^{k}\left(\phi_{\beta}\right)\right\}_{1 \leq k<k\left(\phi_{\beta}\right)}$ is not an invariant under the Markov move of type II and its inverse, where $k\left(\phi_{\beta}\right)$ is the minimal $k$ for $E_{n, j}^{k}\left(\phi_{\beta}\right)=E_{n, j}^{\infty}\left(\phi_{\beta}\right)$. This phenomena corresponds to the quantum effect by the Markov move of type II and its inverse in the symplectic Floer theory. All the higher differentials $d^{k}$ in the spectral sequence count the one dimensional moduli spaces of $J$-holomorphic curves with larger energy. So the Markov move of type II and its inverse will induce a spectral sequence homomorphism (actually isomorphism) for large $k$, in particular $E^{\infty}$-term is invariant under the Markov move of type II and its inverse (see [15]). In [19, 22], these filtered information has been discussed for monotone symplectic manifolds and monotone Lagrangians.

Corollary 2.16. For $j \in \mathbb{Z}_{2 N}$,

$$
\sum_{k \in Z} I_{j+(2 N) k}^{(r)}\left(\phi_{\beta}\right)=H F_{j}^{s y m}\left(\phi_{\beta}\right)
$$

if and only if all the differentials $d^{k}$ in the spectral sequence $\left(E_{n, j}^{k}\left(\phi_{\beta}\right), d^{k}\right)$ are trivial (i.e. $\left.k\left(\phi_{\beta}\right)=1\right)$. In particular, the $\mathbb{Z}$-graded symplectic Floer homology of the braid $\beta$ is a knot invariant if $k\left(\phi_{\beta}\right)=1$.

In general, we see that

$$
\sum_{k \in \mathbb{Z}} I_{j+(2 N) k}^{(r)}\left(\phi_{\beta}\right) \neq H F_{j}^{\mathrm{sym}}\left(\phi_{\beta}\right)
$$

for $j \in \mathbb{Z}_{2 N}$ for $\phi_{\beta}$. The $\mathbb{Z}$-graded symplectic Floer homology $I_{*}^{(r)}\left(\phi_{\beta}\right)$ of the braid $\beta$ can be thought as an integer lift of the symplectic Floer homology $H F_{*}^{\text {sym }}\left(\phi_{\beta}\right)$ of the knot $K=\bar{\beta}$.

Corollary 2.17. The Euler characteristic of the spectral sequence is given by

$$
\chi\left(E_{*, *}^{k}\left(\phi_{\beta}\right)\right)=\chi\left(I_{*}^{(r)}\left(\phi_{\beta}\right)\right)=\chi\left(H F_{*}^{s y m}\left(\phi_{\beta}\right)\right)=\frac{1}{2} \operatorname{sign}(K) .
$$

Proof: Note that the Euler characteristic of the chain complex is same as the Euler characteristic of its induced homology. So the Euler characteristic $\chi\left(E_{*, *}^{k}\left(\phi_{\beta}\right)\right)$ is independent 
of $k$. In particular,

$$
\begin{aligned}
\chi\left(E_{*, *}^{k}\left(\phi_{\beta}\right)\right) & =\chi\left(E_{*, *}^{1}\left(\phi_{\beta}\right)\right)=\chi\left(I_{*}^{(r)}\left(\phi_{\beta}\right)\right) \\
& =\chi\left(E_{*, *}^{\infty}\left(\phi_{\beta}\right)\right)=\chi\left(H F_{*}^{\mathrm{sym}}\left(\phi_{\beta}\right)\right) \\
& =-\lambda_{C L}(K) .
\end{aligned}
$$

Hence the result follows from Corollary 4.3 in [15].

\section{The Symplectic Floer homology of Composite Knots}

3.1. The filtration of the composite knots. We first recall the construction of composite knots. Let $K_{j}(j=1,2)$ be a knot in $S^{3}$. There is a neighborhood $U$ of $p_{j} \in K_{j}$ in $S^{3}$ such that the pair $\left(U, U \cap K_{j}\right)$ is topologically equivalent to the canonical ball pair $\left(B_{j}^{3}, B_{j}^{1}\right)$. By removing the resulting pairs $\left(B_{j}^{3}, B_{j}^{1}\right)$ from $\left(S^{3}, K_{j}\right)$ and sewing the resulting pairs by a homeomorphism $f:\left(\partial B_{2}^{3}, \partial B_{2}^{1}\right) \rightarrow\left(\partial B_{1}^{3}, \partial B_{1}^{1}\right)$, we form the pair connected sum $\left(S^{3}, K_{1}\right) \#\left(S^{3}, K_{2}\right)=\left(S^{3}, K_{1}\right) \cup_{f}\left(S^{3}, K_{2}\right)$. In the convention, we write the connected sum as $K=K_{1} \# K_{2}$ for the connected sum of knots or the composite knot of $K_{1}$ and $K_{2}$.

If the knot $K_{j}$ is represented by a closure of a braid element $\beta_{j}$, then the composite knot $K_{1} \# K_{2}$ can be described as the following. If $K_{1}=\overline{\beta_{1}}, \beta_{1} \in B_{n}$ and $K_{2}=\overline{\beta_{2}}, \beta_{2} \in B_{m}$, then

$$
K_{1} \# K_{2}=\overline{\beta_{1} \Sigma^{n-1}\left(\beta_{2}\right)}, \quad \beta_{1} \Sigma^{n-1}\left(\beta_{2}\right) \in B_{n+m},
$$

where $\Sigma$ is the shift map on the inductive limit of the $B_{n}$ 's and $\Sigma\left(\sigma_{i}\right)=\sigma_{i+1}$. For example, the composite knot of the figure $8 K_{1}=\overline{\sigma_{1} \sigma_{2}^{-1} \sigma_{1} \sigma_{2}^{-1}}$ and the trefoil $K_{2}=\overline{\sigma_{1}^{3}}$ is $\bar{\beta}$, where $\beta=\sigma_{1} \sigma_{2}^{-1} \sigma_{1} \sigma_{2}^{-1} \Sigma^{3-1}\left(\sigma_{1}^{3}\right)=\sigma_{1} \sigma_{2}^{-1} \sigma_{1} \sigma_{2}^{-1} \sigma_{3}^{3}$ (see [四).

Let $K_{1}$ and $K_{2}$ be oriented knots in $S^{3}$, and let $K=K_{1} \# K_{2}$ be their connected sum. Let $\mu_{j} \in \pi_{1}\left(S^{3} \backslash K_{j}\right)(j=1,2)$ be the meridian. By Seifert-Van Kampen's theorem, we have

$$
\pi_{1}\left(S^{3} \backslash K\right)=\pi_{1}\left(S^{3} \backslash K_{1}\right) *_{f} \pi_{1}\left(S^{3} \backslash K_{2}\right),
$$

where the amalgamation homomorphism $f:\left\langle\mu_{1}\right\rangle \rightarrow\left\langle\mu_{2}\right\rangle$ is given by $f\left(\mu_{1}\right)=\mu_{2}$. Let $\mu=\mu_{1}=\mu_{2} \in \pi_{1}\left(S^{3} \backslash K\right)$ be the meridian of $K$. With the identification of the meridians, we can identify $\mathcal{R}^{*}\left(S^{3} \backslash K\right)^{[i]}$ in the following.

Proposition 3.1. $\mathcal{R}^{*}\left(S^{3} \backslash K\right)^{[i]}$ is a disjoint union of

$$
\begin{aligned}
\mathcal{R}^{*}\left(S^{3} \backslash K_{1}\right)^{[i]} \star s_{K_{2}} & =\left\{\left[\rho_{1} * s_{K_{2}}\right] \in \mathcal{R}^{*}\left(S^{3} \backslash K\right)^{[i]}: s_{K_{2}} \text { reducible }\right\}, \\
s_{K_{1}} \star \mathcal{R}^{*}\left(S^{3} \backslash K_{2}\right)^{[i]} & =\left\{\left[s_{K_{1}} * \rho_{2}\right] \in \mathcal{R}^{*}\left(S^{3} \backslash K\right)^{[i]}: s_{K_{1}} \text { reducible }\right\}, \\
\mathcal{R}^{*}\left(S^{3} \backslash K_{1}\right)^{[i]} \star \mathcal{R}^{*}\left(S^{3} \backslash K_{2}\right)^{[i]} & =\left\{\left[\rho_{1} * \rho_{2}\right] \in \mathcal{R}^{*}\left(S^{3} \backslash K\right)^{[i]}\right\} .
\end{aligned}
$$

where $s_{K_{j}}(j=1,2)$ is the unique reducible representation in $\mathcal{R}\left(S^{3} \backslash K_{j}\right)^{[i]}$. For each $\rho_{j} \in$ $\mathcal{R}^{*}\left(S^{3} \backslash K_{j}\right)^{[i]}, \rho_{1} \star s_{K_{2}}$ and $s_{K_{1}} \star \rho_{2}$ are single points, and $\rho_{1} \star \rho_{2}$ represents a $U(1)$ of inequivalent representation classes. 
Proof: Clearly the three spaces are disjoint. If $\rho \in \mathcal{R}^{*}\left(S^{3} \backslash K\right)^{[i]}$, then we define $\rho_{j}=$ $\left.\rho\right|_{\pi_{1}\left(S^{3} \backslash K_{j}\right)}(j=1,2)$ and write $\rho=\rho_{1} \star \rho_{2}$ with $\rho_{1}\left(\mu_{1}\right)=\rho_{2}\left(\mu_{2}\right)$. Given $\rho_{j} \in \mathcal{R}^{*}\left(S^{3} \backslash K_{j}\right)^{[i]}$ we can form

$$
\rho=\rho_{1} * \rho_{2} \quad \text { if and only if } \rho_{1}\left(\mu_{1}\right)=\rho_{2}\left(\mu_{2}\right) .
$$

If both $\rho_{1}$ and $\rho_{2}$ are reducible, the representation $\rho_{1} \star \rho_{2}$ must be reducible. Hence for $\rho \in \mathcal{R}^{*}\left(S^{3} \backslash K\right)^{[i]}$ the restriction $\rho_{j}$ we defined must have at least one irreducible. For $\rho_{1} \in \mathcal{R}^{*}\left(S^{3} \backslash K_{1}\right)^{[i]}$, there is a unique abelian representation $s_{K_{2}}: \pi_{1}\left(S^{3} \backslash K_{2}\right)$ with $\rho_{1}\left(\mu_{1}\right)=$ $s_{K_{2}}\left(\mu_{2}\right)$ and $\operatorname{tr}\left(s_{K_{2}}\right)=0$. Thus there is a unique way to form $\rho_{1} \star s_{K_{2}}$ and $s_{K_{1}} \star \rho_{2}$ up to conjugacy.

For any $\left(\left[\rho_{1}\right],\left[\rho_{2}\right]\right) \in \mathcal{R}^{*}\left(S^{3} \backslash K_{1}\right)^{[i]} \times \mathcal{R}^{*}\left(S^{3} \backslash K_{2}\right)^{[i]}$, there exists $g \in S U(2)$ such that $g \rho_{2}\left(\mu_{2}\right) g^{-1}=\rho_{1}\left(\mu_{1}\right)$. So $\rho_{1} \star\left(g \rho_{2} g^{-1}\right) \in \mathcal{R}^{*}\left(S^{3} \backslash K\right)^{[i]}$. The set of all cosets $[g] \in S U(2) / \pm 1$ satisfying $g \rho_{2}\left(\mu_{2}\right) g^{-1}=\rho_{1}\left(\mu_{1}\right)$ is a coset of the $U(1)$ subgroup containing $\left[\rho_{2}\left(\mu_{2}\right)\right] \in S U(2) / \pm 1$. Therefore $\left[\rho_{1} \star \rho_{2}\right]$ is parameterized by $U(1)$ of inequivalent classes since $S U(2) / \pm 1$ acts freely on the irreducible representations.

Again there is a unique equivalent class of reducible representations in $\mathcal{R}\left(S^{3} \backslash K\right)^{[i]}$. Such a reducible representation $s_{K}$ restricts to $s_{K_{j}} \in \mathcal{R}\left(S^{3} \backslash K_{j}\right)^{[i]}(j=1,2)$. Denote $s_{K}$ by $s_{K_{1}} \star s_{K_{2}}$. The above result has a natural extension to the representations with fixed trace (see [3, 12, 17]). This is known to Klassen in [13].

By the identification between $\operatorname{Fix}\left(\phi_{\beta}\right)$ and $\mathcal{R}^{*}\left(S^{3} \backslash K\right)^{[i]}$, we see that $\mathcal{R}^{*}\left(S^{3} \backslash K\right)^{[i]}$ are critical points of $a_{H}$ on $\Omega_{\phi_{\beta}}$, where $\Omega_{\phi_{\beta}}$ is the space of contractible path $\gamma: \mathbb{R} \rightarrow \mathcal{R}^{*}\left(S^{2} \backslash K\right)^{[i]}$ with $\gamma(s+1)=\phi_{\beta}(\gamma(s))$ and $\beta=\beta_{1} \Sigma^{n-1}\left(\beta_{2}\right)$.

We define a nondegenerate critical submanifold as follows.

Definition 3.2. A manifold $M$ is a nondegenerate critical submanifold of $a_{H}$ on $\Omega_{\phi_{\beta}}$ if (i) $d a_{H}(x)=0$ for every $x \in M$; (ii) $T_{x} M=\operatorname{ker} \operatorname{Hess}\left(a_{H}\right)(x)$; (iii) the Maslov index $\mu(x)$ $(\bmod 2 N)$ is constant on $M$.

Comparing with [2], we only have the relative index for each critical point in $M$. In our case, the negative normal bundle of the critical submanifold is infinite dimensional. The well-defined relative index (iii) can be used to replace the constant Morse index for the critical submanifold. Condition (ii) says that the manifold $M$ is nondegenerate in the normal direction.

Proposition 3.3. The $U(1)$ manifold $\left[\rho_{1} \star \rho_{2}\right]$ is a nondegenerate critical submanifold of $a_{H}$ on $\Omega_{\phi_{\beta}}$ in the normal direction.

Proof: For any element $\rho_{1} \star\left(g \rho_{2} g^{-1}\right) \in \mathcal{R}^{*}\left(S^{3} \backslash K\right)^{[i]}$, we have $d a_{H}\left(\rho_{1} \star\left(g \rho_{2} g^{-1}\right)\right)=0$ for all $g \in S U(2) / \pm 1$ with $\rho_{1}\left(\mu_{1}\right)=\left(g \rho_{2} g^{-1}\right)\left(\mu_{2}\right)$. I.e., every point in $\left[\rho_{1} \star \rho_{2}\right] \cong U(1)$ is a critical point. The Hessian of $a_{H}$ at $\rho_{g}=\rho_{1} \star\left(g \rho_{2} g^{-1}\right)$ is a self-adjoint operator

$$
A_{\rho_{g}}=J \nabla_{\rho_{g}}: L_{1}^{2}\left(\rho_{g}^{*} T \mathcal{R}^{*}\left(S^{2} \backslash K\right)^{[i]}\right) \rightarrow L^{2}\left(\rho_{g}^{*} T \mathcal{R}^{*}\left(S^{2} \backslash K\right)^{[i]}\right) .
$$


By the deformation theory, the space $T_{\rho} \mathcal{R}^{*}\left(S^{2} \backslash K\right)^{[i]}$ (the group cohomology of $\pi_{1}\left(S^{2} \backslash K\right)$ with twisted coefficients) can be identified with the $\rho^{[i]}$-twisted group cohomology $H^{1}\left(S^{2} \backslash\right.$ $\left.K, \rho^{[i]}\right)$ of $S^{2} \backslash K=S^{2} \backslash\left(S^{2} \cap K\right)$ since $S^{2} \backslash K$ is a $K(\pi, 1)$-space. Note that on the smooth part $\mathcal{R}^{*}\left(S^{2} \backslash K\right)^{[i]}$ the Zariski tangent space is the tangent space $T_{\rho} \mathcal{R}^{*}\left(S^{2} \backslash K\right)^{[i]}$. For $\alpha \in H^{1}\left(S^{2} \backslash K, \rho^{[i]}\right)$, then $\alpha$ is a tangent to a path in the representation variety since all the obstructions (cup product and higher Massey products) vanish. So the kernel of $\operatorname{ker} A_{\rho_{g}}$ is ker $A_{\rho_{g}}=B^{1}\left(S^{2} \backslash K, \rho_{g}^{[i]}\right)$ the coboundary of 1-cochains in the twisted group cohomology. For any $g=e^{u_{0}}$, we define

$$
\rho_{t}=\rho_{1} \star e^{-t u_{0}+O\left(t^{2}\right)} \rho_{2} e^{t u_{0}+O\left(t^{2}\right)} .
$$

This provides an adjoint orbit containing $\rho_{g}$, and the cocycle corresponding to $\rho_{t}$ is

$$
u=A d \rho_{g} u_{0}-u_{0} .
$$

So $u$ is the coboundary $\delta u_{0}$. For any path $g_{t}=e^{t v_{0}}, \rho_{t}=g_{t}^{-1}\left(\rho_{g}\right) g_{t}$, the cocycle corresponding to the $\rho_{t}$ is $A d \rho_{g} v_{0}-v_{0}$. On the other hand, $B^{1}\left(S^{2} \backslash K, \rho_{g}^{[i]}\right)$ is the tangent space of the adjoint orbit through $\rho_{g}$.

$$
B^{1}\left(S^{2} \backslash K, \rho_{g}^{[i]}\right)=T_{\rho_{g}}\left[\rho_{1} \star \rho_{2}\right] .
$$

Therefore we obtain that ker $A_{\rho_{g}}=T_{\rho_{g}}\left[\rho_{1} \star \rho_{2}\right]$. So $A_{\rho_{g}}$ is nondegenerate along the normal bundle of $\left[\rho_{1} \star \rho_{2}\right]$ in $\Omega_{\phi_{\beta}}$. Note that $\rho_{1} \star\left(g \rho_{2} g^{-1}\right)$ is equivalent to $\left(g^{-1} \rho_{1} g\right) \star \rho_{2}$. Hence the proof is complete.

Since the symplectic manifold $\mathcal{R}^{*}\left(S^{2} \backslash K\right)^{[i]}$ is monotone by Lemma 2.3 in [15], so we have

$$
\alpha=\frac{\alpha_{1} N\left(K_{1}\right)+\alpha_{2} N\left(K_{2}\right)}{N(K)},
$$

where $N(K)=$ g.c.d $\left(N\left(K_{1}\right), N\left(K_{2}\right)\right)$ and $a_{H}: \Omega_{\phi_{\beta}} \rightarrow \mathbb{R} / \alpha 2 N \mathbb{Z}$ is well-defined. Next we verify the additivity for the Maslov index.

Proposition 3.4. For $r_{j} \in \mathbb{R}_{\phi_{\beta_{j}}}$ and $\rho_{j} \in \mathcal{R}\left(S^{3} \backslash K_{j}\right)^{[i]}(j=1,2)$, we have

$$
\begin{aligned}
& \mu_{K}\left(\rho_{10}^{\left(r_{1}\right)} \star \rho_{2}^{\left(r_{2}\right)}, \rho_{11}^{\left(r_{1}\right)} \star \rho_{2}^{\left(r_{2}\right)}\right)=\mu_{K_{1}}\left(\rho_{10}^{\left(r_{1}\right)}, \rho_{11}^{\left(r_{1}\right)}\right) . \\
& \mu_{K}\left(\rho_{1}^{\left(r_{1}\right)} \star \rho_{20}^{\left(r_{2}\right)}, \rho_{1}^{\left(r_{1}\right)} \star \rho_{21}^{\left(r_{2}\right)}\right)=\mu_{K_{2}}\left(\rho_{20}^{\left(r_{2}\right)}, \rho_{21}^{\left(r_{2}\right)}\right) .
\end{aligned}
$$

Proof: Let $u$ be a path in $\tilde{\Omega}_{\phi_{\beta}}: u(t, s) \in \mathcal{R}\left(S^{2} \backslash K\right)^{[i]},(t, s) \in I \times I$ such that $u(t, s+1)=$ $\phi_{\beta}(u(t, s))$ for each $t \in[0,1]$, and $u(0, s)=\rho_{1}^{\left(r_{1}\right)} \star \rho_{20}^{\left(r_{2}\right)}, u(1, s)=\rho_{1}^{\left(r_{1}\right)} \star \rho_{21}^{\left(r_{2}\right)}$. By the very definition of the Maslov index, we have

$$
\mu_{K}\left(\rho_{1}^{\left(r_{1}\right)} \star \rho_{20}^{\left(r_{2}\right)}, \rho_{1}^{\left(r_{1}\right)} \star \rho_{21}^{\left(r_{2}\right)}\right)=\mu_{u}(\partial(I \times I)),
$$

the evaluation of the Maslov class in $\mathcal{R}\left(S^{2} \backslash K\right)^{[i]}$ on the boundary of $I \times I$. It is deformation invariance rel. end points (see 回, [] $)$. The Maslov index $\mu_{K}\left(\rho_{1}^{\left(r_{1}\right)} \star \rho_{20}^{\left(r_{2}\right)}, \rho_{1}^{\left(r_{1}\right)} \star \rho_{21}^{\left(r_{2}\right)}\right)$ is an integral lifting of the one in $\Omega_{\phi_{\beta}}$. 
For each $t, u(t, \cdot)$ is a contractible path in $\mathcal{R}\left(S^{2} \backslash K\right)^{[i]}:\{t\} \times I \rightarrow \mathcal{R}\left(S^{2} \backslash K\right)^{[i]}$. In particular, there is $u_{j}(t, \cdot)$ is a contractible path in $\mathcal{R}\left(S^{2} \backslash K_{j}\right)^{[i]}$ such that $u_{1}(t, \cdot)=g(t) u_{2}(t, \cdot) g(t)^{-1}$ along the meridian and $u(t, \cdot)=u_{1}(t, \cdot) \star u_{2}(t, \cdot)$. So $u_{1}(t, s)$ satisfies $u_{1}(t, s+1)=$ $\phi_{\beta_{1}}\left(u_{1}(t, s)\right), u_{1}(0, s)=u_{1}(1, s)=\rho_{1}^{\left(r_{1}\right)}$, and $u_{1}(t, \cdot)$ is a contractible path in $\tilde{\mathcal{R}}\left(S^{2} \backslash K_{1}\right)^{[i]}$. Note that there are homotopy equivalent loops corresponding to $\mu_{K_{1}}\left(\rho_{1}, s_{K_{1}}\right)+2 N\left(K_{1}\right) k$. But there is a unique homotopy loop in $\tilde{\mathcal{R}}\left(S^{2} \backslash K_{1}\right)^{[i]}$ corresponding to $\mu_{u_{1}}\left(\rho_{1}^{\left(r_{1}\right)}, \rho_{1}^{\left(r_{1}\right)}\right)=0$. I.e., $u_{1}(t, s)$ on the lifting space $\tilde{\mathcal{R}}\left(S^{2} \backslash K_{1}\right)^{[i]}$ is contractible to a constant loop $\tilde{u}_{1}=\rho_{1}^{\left(r_{1}\right)}$ since $\mu_{K_{1}}\left(\rho_{1}^{\left(r_{1}\right)}, \rho_{1}^{\left(r_{1}\right)}\right)=0$ by Lemma 2.5 . Over the space $\mathcal{R}\left(S^{2} \backslash K_{1}\right)^{[i]}$, we have infinitely many inequivalent loops at $\rho_{1}$ with $\mu\left(\rho_{1}^{\left(r_{1}\right)}, \rho_{1}^{\left(r_{1}\right)}\right) \equiv 0\left(\bmod 2 N\left(K_{1}\right)\right)$. For the unique lift$\operatorname{ing} \rho_{1}^{\left(r_{1}\right)}$, we obtain only one homotopy class for $\mu_{u_{1}}\left(\rho_{1}^{\left(r_{1}\right)}, \rho_{1}^{\left(r_{1}\right)}\right)=0$. Let $U_{1}(t, \tau)$ be the homotopy of $u_{1}$ and $\tilde{u}_{1}$. Thus $U_{1}(t, \tau)\left(\mu_{1}\right)$ parameterizes $g(t) u_{2}(t, \cdot) g(t)^{-1}\left(\mu_{2}\right)$ to $\rho_{2}^{\left(r_{2}\right)}\left(\mu_{2}\right)$. Since $S^{1}$ is path connected, there exists $g(t, \tau)$ such that $U(t, \tau)=U_{1}(t, \tau) \star u_{2}(t, \cdot)$ with $U_{1}(t, \tau)\left(\mu_{1}\right)=g(t, \tau) u_{2}(t, \cdot) g(t, \tau)^{-1}\left(\mu_{2}\right)$ provides a homotopy from $u$ to $\tilde{u}_{1} \star u_{2}$ in the space $\mathcal{R}\left(S^{2} \backslash K\right)^{[i]}$. By the invariance of Maslov index, we have $\mu_{U(t, 0)}=\mu_{U(t, 1)}$, i.e., $\mu_{u}=\mu_{\tilde{u}_{1} \star u_{2}}$. The pullback $\left(\tilde{u}_{1} \star u_{2}\right)^{*} T\left(\mathcal{R}^{*}\left(S^{2} \backslash K\right)^{[i]}\right)$ over $\partial(I \times I)$ is the fiber product of a trivial Lagrangian on $\mathcal{R}^{*}\left(S^{2} \backslash K_{1}\right)^{[i]}$ with a loop of Lagrangians from $\mathcal{R}^{*}\left(S^{2} \backslash K_{2}\right)^{[i]}$ with the proper lifting property. By the Maslov class $\mu$,

$$
\begin{aligned}
\mu_{u}(\partial(I \times I)) & =\mu_{\tilde{u}_{1} \star u_{2}}(\partial(I \times I)) \\
& =\mu_{u_{2}}(\partial(I \times I)) \\
& =\mu_{K_{2}}\left(\rho_{20}^{\left(r_{2}\right)}, \rho_{21}^{\left(r_{2}\right)}\right) .
\end{aligned}
$$

The other identity follows from the same argument.

Note that the monotone symplectic manifold $\mathcal{R}^{*}\left(S^{2} \backslash K\right)^{[i]}$ is not a product of the manifolds $\mathcal{R}^{*}\left(S^{2} \backslash K_{1}\right)^{[i]}$ and $\mathcal{R}^{*}\left(S^{2} \backslash K_{2}\right)^{[i]}$. So one can not apply the catenation property directly (for the catenation property of the Maslov index see 《). Note that the singularity of the respresentaion space has codimension bigger than 2, our Maslov index discussion can be proved in the space $H_{n}$ (see [15, 20] for the notation). Over the space $H_{n} \times H_{m}$ one can apply the catenation property of the Maslov index.

Corollary 3.5. For $r_{j} \in \mathbb{R}_{\phi_{\beta_{j}}}$ and $\rho_{j} \in \mathcal{R}^{*}\left(S^{3} \backslash K_{j}\right)^{[i]}(j=1,2)$, we have

$$
\begin{aligned}
\mu_{K}\left(\rho_{1}^{\left(r_{1}\right)} \star s_{K_{2}}\right) & =\mu_{K_{1}}\left(\rho_{1}^{\left(r_{1}\right)}\right), \\
\mu_{K}\left(s_{K_{1}} \star \rho_{2}^{\left(r_{2}\right)}\right) & =\mu_{K_{2}}\left(\rho_{2}^{\left(r_{2}\right)}\right), \\
\mu_{K}\left(\rho_{1}^{\left(r_{1}\right)} \star \rho_{2}^{\left(r_{2}\right)}\right) & =\mu_{K_{1}}\left(\rho_{1}^{\left(r_{1}\right)}\right)+\mu_{K_{2}}\left(\rho_{2}^{\left(r_{2}\right)}\right) .
\end{aligned}
$$


Proof: Note that the Malsov index on $\mathcal{R}^{*}\left(S^{2} \backslash K\right)^{[i]}$ is calculated with respect to the unique reducible representation $s_{K_{1}} \star s_{K_{2}}=s_{K}$. So we have

$$
\begin{aligned}
\mu_{K}\left(\rho_{1}^{\left(r_{1}\right)} \star s_{K_{2}}\right) & =\mu_{K}\left(\rho_{1}^{\left(r_{1}\right)} \star s_{K_{2}}, s_{K_{1}} \star s_{K_{2}}\right) \\
& =\mu_{K_{1}}\left(\rho_{1}^{\left(r_{1}\right)}, s_{K_{1}}\right) \\
& =\mu_{K_{1}}\left(\rho_{1}^{\left(r_{1}\right)}\right) .
\end{aligned}
$$

Similarly we obtain the following.

$$
\begin{aligned}
\mu_{K}\left(\rho_{1}^{\left(r_{1}\right)} \star \rho_{2}^{\left(r_{2}\right)}\right) & =\mu_{K}\left(\rho_{1}^{\left(r_{1}\right)} \star \rho_{2}^{\left(r_{2}\right)}, s_{K_{1}} \star s_{K_{2}}\right) \\
& =\mu_{K}\left(\rho_{1}^{\left(r_{1}\right)} \star \rho_{2}^{\left(r_{2}\right)}, s_{K_{1}} \star \rho_{2}^{\left(r_{2}\right)}\right)+\mu_{K}\left(s_{K_{1}} \star \rho_{2}^{\left(r_{2}\right)}, s_{K_{1}} \star s_{K_{2}}\right) \\
& =\mu_{K_{1}}\left(\rho_{1}^{\left(r_{1}\right)}\right)+\mu_{K_{2}}\left(\rho_{2}^{\left(r_{2}\right)}\right) .
\end{aligned}
$$

The second equality follows from the path additivity of the Maslov index (see [4]), and the last equality from Proposition 3.4 and the previous two identity.

Corollary 3.6. For each $g \in U(1)$ and $\rho_{j} \in \mathcal{R}\left(S^{3} \backslash K_{j}\right)^{[i]}(j=1,2)$, we have

$$
\mu_{K}\left(\rho_{1} \star \rho_{2}\right) \equiv \mu_{K}\left(\rho_{1} \star_{g} \rho_{2}\right) \equiv \mu_{K_{1}}\left(\rho_{1}\right)+\mu_{K_{2}}\left(\rho_{2}\right) \quad(\bmod 2 N(K)) .
$$

Proof: The proof follows the same argument from Proposition 3.4 with $\left(\bmod 2 N\left(K_{j}\right)\right)$. So we get

$$
\begin{aligned}
\mu_{K}\left(\rho_{1} \star_{g} \rho_{2}\right) & \equiv \mu_{K_{1}}\left(\rho_{1}^{\left(r_{1}\right)}\right) \quad\left(\bmod 2 N\left(K_{1}\right)\right)+\mu_{K_{2}}\left(\rho_{2}^{\left(r_{2}\right)}\right) \quad\left(\bmod 2 N\left(K_{2}\right)\right) \\
& \equiv \mu_{K_{1}}\left(\rho_{1}\right)+\mu_{K_{2}}\left(\rho_{2}\right) \quad(\bmod 2 N(K)) .
\end{aligned}
$$

Hence it is independent of the element $g \in U(1)$.

By Proposition 3.3 and Corollary 3.6, we have showed that the $U(1)$-critical submanifold $\left[\rho_{1} \star \rho_{2}\right]$ is a nondegenerate critical submanifold in the sense of Definition 3.2 for $\rho_{j} \in \mathcal{R}^{*}\left(S^{3} \backslash\right.$ $\left.K_{j}\right)^{[i]}(j=1,2)$. By Proposition 3.4, we see that $\left[\rho_{1}^{\left(r_{1}\right)} \star \rho_{2}^{\left(r_{2}\right)}\right]$ is also a nondegenerate critical submanifold in the sense of Definition 3.2 for the $\mathbb{Z}$-graded symplectic Floer homology.

Definition 3.7. The integral chain complex $C_{n}^{\left(r_{1}, r_{2}\right)}\left(\phi_{\beta_{1} \Sigma^{n-1}\left(\beta_{2}\right)}\right)$ of the braid $\beta_{1} \Sigma^{n-1}\left(\beta_{2}\right)$ with $K=K_{1} \# K_{2}=\overline{\beta_{1} \Sigma^{n-1}\left(\beta_{2}\right)}$ is defined to be

$$
\begin{gathered}
C_{n}^{\left(r_{1}, r_{2}\right)}\left(\phi_{\beta_{1} \Sigma^{n-1}\left(\beta_{2}\right)}\right)=\left\{\rho_{1} \star \rho_{2} \in \operatorname{Fix}\left(\phi_{\beta}\right)=\mathcal{R}^{*}\left(S^{3} \backslash K\right)^{[i]} \mid \mu_{K}\left(\rho_{1}^{\left(r_{1}\right)} \star \rho_{2}^{\left(r_{2}\right)}\right)=n\right. \\
\text { and } \tilde{a}_{H_{j}}\left(\rho_{j}^{\left(r_{j}\right)}\right) \in\left(r_{j}, r_{j}+2 \alpha_{j} N\left(K_{j}\right), r_{j} \in \mathbb{R}_{\phi_{\beta_{j}}}\right\} .
\end{gathered}
$$

Note that $\operatorname{Fix}\left(\phi_{\beta}\right)\left(\beta=\beta_{1} \Sigma^{n-1}\left(\beta_{2}\right)\right)$ consists of $\rho_{1} \star s_{K_{2}}, s_{K_{1}} \star \rho_{2}$ and $\rho_{1} \star \rho_{2}$. There is an one-to-one correspondence between the elements in Fix $\left(\phi_{\beta}\right)$ and the elements in $\left\{\rho_{1}^{\left(r_{1}\right)} \star \rho_{2}^{\left(r_{2}\right)} \mid \rho_{j}^{\left(r_{j}\right)} \in \mathcal{R}\left(S^{3} \backslash K_{j}\right)^{[i]}, \rho_{1} \star \rho_{2} \in \mathcal{R}^{*}\left(S^{3} \backslash K\right)^{[i]}, \tilde{a}_{H_{j}}\left(\rho_{j}^{\left(r_{j}\right)}\right) \in\left(r_{j}, r_{j}+2 \alpha_{j} N\left(k_{j}\right)\right)\right\}$. By Proposition 3.4, we have that Definition 3.7 for the composite knot is well-defined. Fixing the symplectic action with respect to the $r_{j} \in \mathbb{R}_{\phi_{\beta_{j}}}$ is necessary. Since there are $g_{j, k} \in$ 
$\pi_{1}\left(\tilde{\Omega}_{\phi_{\beta_{j}}}\right)$ with $\tilde{a}_{H_{j}}\left(g_{j, k} \rho_{j}^{\left(r_{j}\right)}\right) \in\left(r_{j}+2 \alpha_{j} k N\left(K_{j}\right), r_{j}+2 \alpha_{j}(k+1) N\left(K_{j}\right)\right)$ and $\mu_{K_{j}}\left(g_{j, k} \rho_{j}^{\left(r_{j}\right)}\right)=$ $\mu_{K_{j}}\left(\rho_{j}^{\left(r_{j}\right)}\right)+2 k N\left(K_{j}\right)$, we have

$$
\begin{aligned}
\mu_{K}\left(g_{1, k N_{2}} \rho_{1}^{\left(r_{1}\right)} \star g_{2,-k N_{1}} \rho_{2}^{\left(r_{2}\right)}\right) & =\sum_{j=1}^{2} \mu_{K_{j}}\left(g_{\left.j,(-1)^{j+1} k N_{-j+3} \rho_{j}^{\left(r_{j}\right)}\right)}\right. \\
& =\sum_{j=1}^{2}\left(\mu_{K_{j}}\left(\rho_{j}^{\left(r_{j}\right)}\right)+2\left(k N_{2}\right) N\left(K_{1}\right)+2\left(-k N_{1}\right) N\left(K_{2}\right)\right. \\
& =\sum_{j=1}^{2}\left(\mu_{K_{j}}\left(\rho_{j}^{\left(r_{j}\right)}\right) .\right.
\end{aligned}
$$

So $\mu_{K}\left(g_{1, k N_{2}} \rho_{1} \star g_{2,-k N_{1}} \rho_{2}\right) \equiv \sum_{j=1}^{2} \mu_{K_{j}}\left(\rho_{j}^{\left(r_{j}\right)}\right)(\bmod 2 N(K))$ for infinitely many $k$. I.e., the element $g_{1, k N_{2}} \rho_{1}^{\left(r_{1}\right)} \star g_{2,-k N_{1}} \rho_{2}^{\left(r_{2}\right)}$ has the same Maslov index $n$, but different symplectic actions with respect to $r_{j}(j=1,2)$. Thus our chain groups $C_{*}^{\left(r_{1}, r_{2}\right)}\left(\phi_{\beta}\right)$ is well-defined, finitely generated from $C_{*}^{\left(r_{j}\right)}\left(\phi_{\beta_{j}}\right)(j=1,2)$, and the integral liftings of $C_{*}\left(\phi_{\beta_{1} \Sigma^{n-1}\left(\beta_{2}\right)}\right)$ corresponding to $r_{j} \in \mathbb{R}_{\phi_{\beta_{j}}}(j=1,2)$ is the integral chain groups $C_{*}^{\left(r_{1}, r_{2}\right)}\left(\phi_{\beta_{1} \Sigma^{n-1}\left(\beta_{2}\right)}\right)$. See [18, 19] for more discussions on this kind of formulations.

3.2. Spectral sequences for the composite knots representing by braids. In this subsection, we form a filtered Floer chain complex for the braid $\beta=\beta_{1} \Sigma^{n-1}\left(\beta_{2}\right)$ representing the composite knot $K=K_{1} \# K_{2}$. The filtered chain complex of the braid naturally associates to a spectral sequence. The spectral sequence converges to the $\mathbb{Z}$-graded symplectic Floer homology $I_{*}^{\left(r_{1}, r_{2}\right)}\left(\phi_{\beta}\right)$ and collapses at third term.

For the composite knot $K_{1} \# K_{2}=\overline{\beta_{1} \Sigma^{n-1}\left(\beta_{2}\right)}$ as in $\S 3.1$, we know that the $\mathbb{Z}$-graded symplectic Floer chain groups $C_{*}^{\left(r_{1}, r_{2}\right)}\left(\phi_{\beta}\right)$ are generated freely by

$$
\rho_{1} \star s_{K_{2}}, \quad s_{K_{1}} \star \rho_{2}, \quad \rho_{1} \star \rho_{2} .
$$

The free generated group $C_{*}^{\left(r_{1}, r_{2}\right)}\left(\phi_{\beta}\right)=\oplus_{q} C_{q}^{\left(r_{1}, r_{2}\right)}\left(\phi_{\beta}\right)$ is a graded differential group with respect to the boundary map $\partial_{*}^{\left(r_{1}, r_{2}\right)}, \partial C_{q}^{\left(r_{1}, r_{2}\right)}\left(\phi_{\beta}\right) \subset C_{q-1}^{\left(r_{1}, r_{2}\right)}\left(\phi_{\beta}\right)$. Recall the $\mathbb{Z}$-graded symplectic Floer chain group

$$
\begin{gathered}
C_{q}^{\left(r_{1}, r_{2}\right)}\left(\phi_{\beta}\right)=\left\{\rho \in \mathcal{R}^{*}\left(S^{3} \backslash K\right)^{[i]} \mid \mu(\tilde{\rho})=q,\right. \\
\left.\tilde{a}_{H_{j}}\left(\rho_{j}{ }^{\left(r_{j}\right)}\right) \in\left(r_{j}, r_{j}+2 \alpha_{j} N_{j}\right) \text { for } \rho=\rho_{1} \star \rho_{K_{2}} \text { and } \rho=\rho_{1} \star s_{2}\right\} .
\end{gathered}
$$

There is an associated filtration compatible with the grading, defined as follows:

$$
F_{p} C_{*}^{\left(r_{1}, r_{2}\right)}\left(\phi_{\beta}\right)=\bigoplus_{k \leq p}\left\{\mathbb{Z}\langle\rho\rangle \mid \rho \in C_{k}^{\left(r_{1}, r_{2}\right)}\left(\phi_{\beta}\right)\right\} .
$$

The filtration is an increasing filtration $F_{p} C_{q}^{\left(r_{1}, r_{2}\right)}\left(\phi_{\beta}\right) \subset F_{p+1} C_{q}^{\left(r_{1}, r_{2}\right)}\left(\phi_{\beta}\right)$. The filtration (18) is different from the decreasing filtration (2.12). Again the symplectic action functional 
is non-decreasing along the gradient flows ( $J$-holomorphic curves). It follows that the $\mathbb{Z}$ graded symplectic Floer boundary map $\partial^{\left(r_{1}, r_{2}\right)}: F_{p} C_{q}^{\left(r_{1}, r_{2}\right)}\left(\phi_{\beta}\right) \hookrightarrow F_{p} C_{q-1}^{\left(r_{1}, r_{2}\right)}\left(\phi_{\beta}\right)$ preserves the filtration (18). By the standard method in algebraic topology [26], we have a spectral sequence induced from the filtration (18).

Lemma 3.8. For the braid $\beta=\beta_{1} \Sigma^{n-1}\left(\beta_{2}\right)$, we have the $E_{*, *}^{0}$ and $E_{*, *}^{1}$ terms of the filtration (18) given by the following.

$$
\begin{aligned}
& E_{p, q}^{0}=F_{p} C_{q}^{\left(r_{1}, r_{2}\right)}\left(\phi_{\beta}\right) / F_{p-1} C_{q}^{\left(r_{1}, r_{2}\right)}\left(\phi_{\beta}\right)=C_{p}^{\left(r_{1}, r_{2}\right)}\left(\phi_{\beta}\right) ; \\
& E_{p, 0}^{1}=\oplus Z\langle\rho\rangle, \text { where } \rho \in C_{p}^{\left(r_{1}, r_{2}\right)}\left(\phi_{\beta}\right), \quad p=\mu_{K}(\tilde{\rho}) ; \\
& E_{p, q}^{1}=0, \text { for } q \neq 0 \text { and } \rho=\rho_{1} \star s_{K_{2}}, s_{K_{1}} \star \rho_{2} ; \\
& E_{p, 1}^{1}=\oplus Z\left\langle\left(\rho_{1} \star \rho_{2}\right)_{1}\right\rangle, \quad \mu_{K}\left(\rho_{1}^{\left(r_{1}\right)} \star \rho_{2}^{\left(r_{2}\right)}\right)=p ; \\
& E_{p, q}^{1}=0, \quad \text { for } q \neq 0,1, \quad \rho=\rho_{1} \star \rho_{2}, p=\mu_{K}\left(\rho_{1}^{\left(r_{1}\right)} \star \rho_{2}^{\left(r_{2}\right)}\right),
\end{aligned}
$$

where $\left(\rho_{1} \star \rho_{2}\right)_{1}$ is the critical point of the Morse index 1 in $U(1)=\rho_{1} \star \rho_{2}$.

Proof: The $E_{*, *}^{0}$-terms follow from the very definition of spectral sequence induced from the filtration (18). The $E_{*, *}^{1}$-terms follows from all three types of generators and $H_{*}(U(1), \mathbb{Z})$, since $d_{0}$ is the differential of $\rho$ 's for $\rho \in C_{p}^{\left(r_{1}, r_{2}\right)}\left(\phi_{\beta}\right)$. If $\rho=\rho_{1} \star s_{K_{2}}$ and $s_{K_{1}} \star \rho_{2}$, it is a single point so that $E_{p, 0}^{1}=Z\langle\rho\rangle$ for $p=\mu(\tilde{\rho})$ and zero otherwise. If $\rho=\rho_{1} \star \rho_{2}$, then it gives an $U(1)$ component which $d_{0}$ is the boundary map of the standard cellular chain complex of $U(1)$. So the $E_{*, *}^{1}$-terms follows from the Maslov index calculations and the homology of $U(1)$ for each copy of $\rho_{1} \star \rho_{2}$.

One can define a precise perturbation of the symplectic action such that all the critical points are isolated (see [2, 18]). The perturbation keeps the isolated points $\rho_{1} \star s_{K_{2}}$ and $s_{K_{1}} \star \rho_{2}$, and generates a Morse function along the tubular neighborhood of the critical submanifold $\rho_{1} \star \rho_{2}$. This also gives an interpretation of the $E_{*, *}^{1}\left(\phi_{\beta}\right)$ term in Lemma 3.8 .

Theorem 3.9. There is a spectral sequence $\left(E_{p, q}^{r}\left(\phi_{\beta}\right), d_{r}\right)$ determined by the filtration (18) for the braid $\beta=\beta_{1} \Sigma^{n-1}\left(\beta_{2}\right)$ of the composite knot $K_{1} \# K_{2}$. The spectral sequence with $\left(E_{p, q}^{1}\right.$ given by Lemma 3.8)

$$
E_{p, q}^{1}\left(\phi_{\beta}\right) \cong H_{p+q}\left(F_{p} C_{*}^{\left(r_{1}, r_{2}\right)}\left(\phi_{\beta}\right) / F_{p-1} C_{*}^{\left(r_{1}, r_{2}\right)}\left(\phi_{\beta}\right)\right),
$$

converges to the $\mathbb{Z}$-graded symplectic Floer homology $I_{*}^{\left(r_{1}, r_{2}\right)}\left(\phi_{\beta}\right)(* \in \mathbb{Z})$ of the braid $\beta$.

Proof: Because $\mathcal{R}^{*}\left(S^{3} \backslash \bar{\beta}\right)^{[i]}$ is compact, so $E_{p, q}^{1}\left(\phi_{\beta}\right)$ is finitely generated by Lemma 3.8. In particular the filtration is bounded. Since $\bigcup_{p} F_{p} C_{*}^{\left(r_{1}, r_{2}\right)}\left(\phi_{\beta}\right)=C_{*}^{\left(r_{1}, r_{2}\right)}\left(\phi_{\beta}\right)$ (the filtration is exhaustive), we get a convergent spectral sequence by the result in [26]. By the definition of the filtration $F_{p} C_{*}^{\left(r_{1}, r_{2}\right)}\left(\phi_{\beta}\right)$, the higher differentials calculate all the integral Floer boundary map $\partial_{\phi_{\beta}}^{\left(r_{1}, r_{2}\right)}$ (see [9, 18, 19]). Thus the spectral sequence converges to the $\mathbb{Z}$-graded symplectic Floer homology of the braid $\beta$. 
Proposition 3.10. The spectral sequence $\left(E_{*, *}^{r}\left(\phi_{\beta}\right), d_{r}\right)$ in Theorem 3.9 collapses at the third term. Thus the term $E_{*, *}^{3}\left(\phi_{\beta}\right)=E_{*, *}^{\infty}\left(\phi_{\beta}\right)$ gives the $\mathbb{Z}$-graded symplectic Floer homology $I_{*}^{\left(r_{1}, r_{2}\right)}\left(C_{*}^{\left(r_{1}, r_{2}\right)}\left(\phi_{\beta}\right)\right)$ of the braid $\beta=\beta_{1} \Sigma^{n-1}\left(\beta_{2}\right)$.

Proof: For the differential $d_{r}: E_{p, q}^{r}\left(\phi_{\beta}\right) \rightarrow E_{p-r, q+r-1}^{r}\left(\phi_{\beta}\right), r \geq 3$, we have $E_{p, q}^{r}\left(\phi_{\beta}\right)=0$ for $q<0$ or $q>1$ by Lemma 3.8. The differential $d_{r}$ has either source zero or target zero. So $d_{r}=0$ for all $r \geq 3$. Hence the spectral sequence collapses at the third term.

By Theorem 2.14, we can form another spectral sequence from $I_{*}^{\left(r_{1}, r_{2}\right)}\left(\phi_{\beta}\right)$ which con-

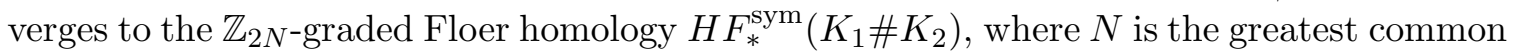
divisor of $N\left(K_{1}\right)$ and $N\left(K_{2}\right)$.

In order to make some computations of $H F_{*}^{\mathrm{sym}}\left(K_{1} \# K_{2}\right)$, we need to describe all the differentials in Theorem 3.9 and combine the differentials in Theorem 2.14. By Lemma 3.10, we know that there are only two possibly nontrivial differentials $d_{1}$ and $d_{2}$. In the section 4 , we give a description of $d_{1}$. We do not know how to characterize the differential $d_{2}$ yet. We leave it to a future study.

We define the associated Poincaré-Laurent polynomial for the spectral sequence by

$$
P_{K}^{(r)}\left(E_{*, *}^{k}, t\right)=\sum_{n \in \mathbb{Z}}\left(\operatorname{dim}_{\mathbb{Z}} E_{n, j}^{k}\right) t^{n} .
$$

By Theorem 2.14, $P_{K}^{(r)}\left(E_{*, *}^{k}, t\right)=\sum_{n \in \mathbb{Z}}\left(\operatorname{dim}_{\mathbb{Z}} I_{n}^{(r)}\left(\phi_{\beta}\right)\right) t^{n}$.

Proposition 3.11. The following identities hold.

$$
\begin{gathered}
P_{K}^{(r)}\left(E_{*, *}^{k}, t\right)=\left(1+t^{2 N k-1}\right) P_{K}^{(r)}\left(B_{*, *}^{k}, t\right)+P_{K}^{(r)}\left(E_{*, *}^{k+1}, t\right) . \\
P_{K}^{(r)}\left(E_{*, *}^{1}, t\right)=\sum_{i=1}^{k}\left(1+t^{2 N i-1}\right) P_{K}^{(r)}\left(B_{*, *}^{i}, t\right)+P_{K}^{(r)}\left(H F_{*}^{s y m}, t\right) .
\end{gathered}
$$

Proof: Let $Z_{n, j}^{k}=\operatorname{ker}\left\{d^{k}: E_{n, j}^{k} \rightarrow E_{n+2 N k-1, j-1}^{k}\right\}$ and $B_{n, j}^{k}=\operatorname{Im} d^{k} \cap E_{n, j}^{k}$. There are two short exact sequences:

$$
\begin{gathered}
0 \rightarrow Z_{n, j}^{k} \rightarrow E_{n, j}^{k} \rightarrow B_{n+2 N k-1, j-1}^{k} \rightarrow 0 \\
0 \rightarrow B_{n, j}^{k} \rightarrow Z_{n, j}^{k} \rightarrow E_{n, j}^{k+1} \rightarrow 0 .
\end{gathered}
$$

So the degree $2 N k-1$ of $d^{k}$ derives the following identity.

$$
P_{K}^{(r)}\left(E_{*, *}^{k}, t\right)=\left(1+t^{2 N k-1}\right) P_{K}^{(r)}\left(B_{*, *}^{k}, t\right)+P_{K}^{(r)}\left(E_{*, *}^{k+1}, t\right) .
$$

Note that the spectral sequence converges at finite steps. So the identity (21) follows from by iterating the identity (20) and using Theorem 2.14. 
Note that $\chi_{K}\left(E_{*, *}^{k}\left(\phi_{\beta}\right)\right)=P_{K}^{(r)}\left(E_{*, *}^{k}\left(\phi_{\beta}\right),-1\right)$ and Corollary 2.17 is an easy consequence of Proposition 3.11. By Lemma 3.8 and Theorem 3.9, we obtain

$$
\chi_{K_{1} \# K_{2}}\left(E_{*, *}^{1}\left(\phi_{\beta}\right)\right)=\chi_{K_{1}}\left(E_{*, *}^{1}\left(\phi_{\beta_{1}}\right)\right)+\chi_{K_{2}}\left(E_{*, *}^{1}\left(\phi_{\beta_{2}}\right)\right) .
$$

By Corollary 2.17 and (22),

$$
\chi_{K_{1} \# K_{2}}\left(E_{*, *}^{k}\left(\phi_{\beta}\right)\right)=\chi_{K_{1}}\left(E_{*, *}^{k}\left(\phi_{\beta_{1}}\right)\right)+\chi_{K_{2}}\left(E_{*, *}^{k}\left(\phi_{\beta_{2}}\right)\right) .
$$

In particular, we have the equality on the $E^{\infty}$-term:

$$
\chi_{K_{1} \# K_{2}}\left(H F_{*}^{\mathrm{sym}}\left(\phi_{\beta}\right)\right)=\chi_{K_{1}}\left(H F_{*}^{\mathrm{sym}}\left(\phi_{\beta_{1}}\right)\right)+\chi_{K_{2}}\left(H F_{*}^{\mathrm{sym}}\left(\phi_{\beta_{2}}\right)\right) .
$$

Thus we obtain the classical result

$$
\operatorname{sign}\left(K_{1} \# K_{2}\right)=\operatorname{sign}\left(K_{1}\right)+\operatorname{sign}\left(K_{2}\right),
$$

as one of identities from our spectral sequence for the symplectic Floer homology of the braid.

\section{Description of $d_{1}$}

We are going to define two special boundary maps which are not used in the definitions of the $\mathbb{Z}$-graded and the $\mathbb{Z}_{2 N}$-graded symplectic Floer homologies in 15 and $\S 2$ of the present paper. These two special boundary maps do contribute for the composite knot. In particular, these two special boundary maps are part of the differential $d_{1}$ in the spectral sequence in Theorem 3.9 and Proposition 3.10. In the gauge theory, we have the same special boundary maps used in the instanton Floer homology of connected sums of two integral homology 3 -spheres [16, 18].

Definition 4.1. $d_{\beta}: C_{1}^{(r)}\left(\phi_{\beta}\right) \rightarrow \mathbb{Z}\left\langle s_{K}\right\rangle$ is defined by

$$
d_{\beta} \rho=\# \hat{\mathcal{M}}^{1}\left(\rho, s_{K}\right) \cdot s_{K},
$$

and $\delta_{\beta}: \mathbb{Z}\left\langle s_{K}\right\rangle \rightarrow C_{-1}^{(r)}\left(\phi_{\beta}\right)$ is defined by

$$
\delta_{Y} s_{K}=\sum_{\rho \in C_{-1}\left(\phi_{\beta}\right)} \# \hat{\mathcal{M}}^{1}\left(s_{K}, \rho\right) \cdot \rho .
$$

Note that by fixing $\mu^{(r)}\left(s_{K}\right)=0$, both maps $d_{\beta}$ and $\delta_{\beta}$ are counting the one dimensional moduli spaces from/to the unique reducible representation $s_{K}$.

Lemma 4.2. $d_{\beta} \partial^{(r)}=0, \quad \partial^{(r)} \delta_{\beta}=0$.

Proof: The proof is similar to the proof that $\partial^{(r)} \circ \partial^{(r)}=0$ in Lemma 2.8 for the $\mathbb{Z}$-graded symplectic Floer chain complex of a braid (also see [6, 15]).

Note that for the Floer boundary map $\partial$ in 15] we also have $d_{\beta} \circ \partial=0$ and $\partial \circ \delta_{\beta}=0$. Let $\partial_{j}^{\left(r_{j}\right)}: C_{*}^{\left(r_{j}\right)}\left(\phi_{\beta_{j}}\right) \rightarrow C_{*-1}^{\left(r_{j}\right)}\left(\phi_{\beta_{j}}\right)(j=1,2)$ be the boundary map in Definition 2.6 of the 
$\mathbb{Z}$-graded symplectic Floer chain complex of the braid $\beta_{j}$. The main result of this section is a description of $d_{1}$ in Theorem 3.9 .

Theorem 4.3. The differential $d_{1}$ of the spectral sequence $\left(E_{p, q}^{r}\left(\phi_{\beta}\right), d_{r}\right)$ in Theorem 3.9 is given by

$$
d_{1}=\partial_{1}^{\left(r_{1}\right)} \star I d_{2} \pm I d_{1} \star \partial_{2}^{\left(r_{2}\right)} \pm d_{\beta_{1}} \star I d_{2} \pm I d_{1} \star d_{\beta_{2}}+\delta_{\beta_{1}} \star I d_{2} \pm I d_{1} \star \delta_{\beta_{2}},
$$

where $\beta=\beta_{1} \Sigma^{n-1}\left(\beta_{2}\right)$ and $\bar{\beta}=K_{1} \# K_{2}$.

Remark: The notation in Theorem 4.3 and the determination of signs can best be explained by two simple examples:

$$
\begin{gathered}
d_{1}\left(\rho_{1} \star \rho_{2}\right)_{1}=\left(\partial_{1}^{\left(r_{1}\right)} \rho_{1} \star \rho_{2}\right)_{1}+(-1)^{\mu\left(\rho_{1}\right)}\left(\rho_{1} \star \partial_{2}^{\left(r_{2}\right)} \rho_{2}\right)_{1}, \\
d_{1}\left(\rho_{1} \star s_{K_{2}}\right)=\partial_{1}^{\left(r_{1}\right)} \rho_{1} \star s_{K_{2}}+(-1)^{\mu\left(\rho_{1}\right)}\left(\rho_{1} \star \delta_{\beta_{2}} s_{K_{2}}\right)_{0} .
\end{gathered}
$$

Note that $\mu\left(\rho_{1}{ }^{\left(r_{1}\right)}\right) \equiv \mu\left(\rho_{1}\right)(\bmod 2 N)$. We have extended our notation here in the obvious way: $\left(\sum m_{j} \rho_{1, j}\right) \star \rho_{2}=\sum m_{j}\left(\rho_{1, j} \star \rho_{2}\right)$. Theorem 4.3 gives a full description of the differential $d_{1}$ in terms of two special boundary maps and the $\mathbb{Z}$-graded boundary maps of $\beta_{1}$ and $\beta_{2}$. The reducible representations $s_{K_{1}}$ and $s_{K_{2}}$ do contribute via the special maps $d_{\beta_{j}}$ and $\delta_{\beta_{j}}$ $(j=1,2)$ in Definition 4.1 .

The differential $d_{1}: E_{p, q}^{1} \rightarrow E_{p-1, q}^{1}$ is possibly nonzero for $q=0$ and $q=1$. Note that $E_{p, q}^{1}$ is generated from $C_{p}^{\left(r_{1}, r_{2}\right)}\left(\phi_{\beta}\right)$ by Lemma 3.8. Hence the differential $d_{1}$ is calculated by the following:

$$
\begin{gathered}
d_{1}\left(\rho_{1} \star \rho_{2}\right)_{i}=\sum \hat{\mathcal{M}}_{J}^{1}\left(\left(\rho_{1} \star \rho_{2}\right)_{i},\left(\rho_{1}^{\prime} \star \rho_{2}^{\prime}\right)_{i}\right) \cdot\left(\rho_{1}^{\prime} \star \rho_{2}^{\prime}\right)_{i}, \quad \text { for } i=0,1 ; \\
d_{1}\left(\rho_{1} \star s_{K_{2}}\right)=\sum \hat{\mathcal{M}}_{J}^{1}\left(\rho_{1} \star s_{K_{2}},\left(\rho_{1}^{\prime} \star \rho_{2}^{\prime}\right)_{0}\right) \cdot\left(\rho_{1}^{\prime} \star \rho_{2}^{\prime}\right)_{0},
\end{gathered}
$$

for the critical points $\left(\rho_{1}^{\prime} \star \rho_{2}^{\prime}\right)_{0}$ and $\left(\rho_{1}^{\prime} \star \rho_{2}^{\prime}\right)_{1}$ of the $U(1)=\rho_{1}^{\prime} \star \rho_{2}^{\prime}$ with Morse index 0 and 1. By (25) and (26), we have

$$
\begin{gathered}
\mu_{K}\left(\left(\rho_{1} \star \rho_{2}\right)_{i}\right)-\mu_{K}\left(\left(\rho_{1}^{\prime} \star \rho_{2}^{\prime}\right)_{i}\right)=1 ; \\
\mu_{K}\left(\rho_{1} \star s_{2}\right)-\mu_{K}\left(\left(\rho_{1}^{\prime} \star \rho_{2}^{\prime}\right)_{0}\right)=1 .
\end{gathered}
$$

By Proposition 3.4, Corollary 3.5 and Lemma 3.8,

$$
\mu_{K}\left(\left(\rho_{1} \star \rho_{2}\right)_{i}\right)=\mu_{K_{1}}\left(\rho_{1}^{\left(r_{1}\right)}\right)+\mu_{K_{2}}\left(\rho_{2}^{\left(r_{2}\right)}\right)+i, \quad i=0,1 .
$$

So the equation (27) is reduced to

$$
\mu_{K}\left(\left(\rho_{1} \star \rho_{2}\right),\left(\rho_{1}^{\prime} \star \rho_{2}\right)\right)+\mu_{K}\left(\left(\rho_{1}^{\prime} \star \rho_{2}\right),\left(\rho_{1}^{\prime} \star \rho_{2}^{\prime}\right)\right)=1,
$$

by Proposition 3.4 and the path additivity of the Maslov index in [4]. By the genericity, the moduli space of $J$-holomorphic curves is empty if the Maslov index is negative over 
the compact monotone symplectic manifolds (see [6, 14, 24]). So we have the following two cases:

$$
\begin{aligned}
& \mu_{K}\left(\left(\rho_{1} \star \rho_{2}\right),\left(\rho_{1}^{\prime} \star \rho_{2}\right)\right)=1, \quad \mu_{K}\left(\left(\rho_{1}^{\prime} \star \rho_{2}\right),\left(\rho_{1}^{\prime} \star \rho_{2}^{\prime}\right)\right)=0, \\
& \mu_{K}\left(\left(\rho_{1} \star \rho_{2}\right),\left(\rho_{1}^{\prime} \star \rho_{2}\right)\right)=0, \quad \mu_{K}\left(\left(\rho_{1}^{\prime} \star \rho_{2}\right),\left(\rho_{1}^{\prime} \star \rho_{2}^{\prime}\right)\right)=1 .
\end{aligned}
$$

For the case $\mu_{K}\left(\left(\rho_{1}^{\prime} \star \rho_{2}\right),\left(\rho_{1}^{\prime} \star \rho_{2}^{\prime}\right)\right)=0$, we have $\mu_{K_{2}}\left(\rho_{2}, \rho_{2}^{\prime}\right)=0$ over $\mathcal{R}^{*}\left(S^{2} \backslash K_{2}\right)^{[i]}$, and $\rho_{2}=\rho_{2}^{\prime}$ by the monotonicity. From the Maslov index calculation, the difference of Maslov index 1 must have the difference of Malsov index 1 on one side and 0 on the other side. Since both symplectic manifolds in the consideration are monotone, so we must have a constant on the one side from the index calculation.

Now we study the moduli space of $\mathcal{M}_{J}\left(\rho_{1} \star \rho_{2}, \rho_{1}^{\prime} \star \rho_{2}\right)$ for the Maslov index $\mu_{K_{1}}\left(\rho_{1}, \rho_{1}^{\prime}\right)=1$. Since the boundary map only counts the 1 -dimensional moduli space, not $1(\bmod 2 N(K))$ dimensional moduli space. For $u \in \mathcal{M}_{J}^{1}\left(\rho_{1} \star \rho_{2}, \rho_{1}^{\prime} \star \rho_{2}\right)$, we have $\mu_{K}(u)=1$. We are going to use a cobordism technique to relate the moduli space $\mathcal{M}_{J}^{1}\left(\rho_{1} \star \rho_{2}, \rho_{1}^{\prime} \star \rho_{2}\right)$ with the moduli space $\mathcal{M}_{J}^{1}\left(\rho_{1}, \rho_{1}^{\prime}\right) \times\left\{\rho_{2}\right\}$.

Let $J$ be an almost complex structure on $\mathcal{R}^{*}\left(S^{2} \backslash K\right)^{[i]}$ which is compatible with the symplectic structure. So $J$ is constructed from an almost complex structure $J_{j}$ on $\mathcal{R}^{*}\left(S^{2} \backslash\right.$ $\left.K_{j}\right)^{[i]}(j=1,2)$. This gives the usual decomposition into $J$-linear and $J$-anti-linear maps

$$
\begin{aligned}
\mathcal{E}_{u} & =\operatorname{Hom}\left(T \mathbb{C}, u^{*} T \mathcal{R}^{*}\left(S^{2} \backslash K\right)^{[i]}\right) \\
& =T^{1,0} \mathbb{C} \otimes_{J} u^{*} T \mathcal{R}^{*}\left(S^{2} \backslash K\right)^{[i]} \oplus T^{0,1} \mathbb{C} \otimes_{J} u^{*} T \mathcal{R}^{*}\left(S^{2} \backslash K\right)^{[i]} .
\end{aligned}
$$

The subbundle $\mathcal{E}^{0,1}$ is given by its fibre

$$
\mathcal{E}_{u}^{0,1}=L^{p}\left(T^{0,1} \mathbb{C} \otimes_{J} u^{*} T \mathcal{R}^{*}\left(S^{2} \backslash K\right)^{[i]}\right) \rightarrow L_{1}^{p}\left(\operatorname{Map}\left(\mathbb{C}, \mathcal{R}^{*}\left(S^{2} \backslash K\right)^{[i]}\right)\right) .
$$

Choose a trivialization of $\mathcal{E}$ which is compatible with $J$. The operation of $J$ on $\mathcal{E}$ appears as an endomorphism of $L^{p}\left(\mathbb{C}, \operatorname{Hom}\left(T \mathbb{C}, u^{*} T \mathcal{R}^{*}\left(S^{2} \backslash K\right)^{[i]}\right)\right)$. The section $u \mapsto \bar{\partial}_{J} u=\frac{1}{2}(d u+J \circ$ $d u \circ i$ ) is the projection of the section $u \mapsto d u$ onto the subbundle $\mathcal{E}^{0,1}$. The corresponding perturbed section $\bar{\partial}_{J, H}$ is smooth, and its zero set is the moduli space $\mathcal{M}_{J}\left(\rho_{1} \star \rho_{2}, \rho_{1}^{\prime} \star \rho_{2}\right)$, where $\bar{\partial}_{J, H} u=\frac{\partial u}{\partial t}+J\left(\frac{\partial u}{\partial s}-X_{s}(u)\right)=0$ and $X_{s}$ is the corresponding Hamiltonian vector field of the Hamiltonian function $H: \mathcal{R}^{*}\left(S^{2} \backslash K\right)^{[i]} \times \mathbb{R} \rightarrow \mathbb{R}$.

Note that the monotone symplectic structure on $\mathcal{R}^{*}\left(S^{2} \backslash K\right)^{[i]}$ is induced from the one on $Q_{n+m}=Q_{n} \times Q_{m}$ since $\beta_{1} \in B_{n}$ and $\beta_{2} \in B_{m}$ (see Lemma 2.2 in [15]). The symplectic structure $\omega$ restricts on the symplectic structure $\omega_{j}$ on $\mathcal{R}^{*}\left(S^{2} \backslash K_{j}\right)^{[i]}(j=1,2)$. For the Hamiltonian function $H: \mathcal{R}^{*}\left(S^{2} \backslash K\right)^{[i]} \times \mathbb{R} \rightarrow \mathbb{R}$, we have $\omega\left(X_{H}, \cdot\right)=d H$ and $\omega_{j}\left(X_{H}, \cdot\right)=$ $\left.d H\right|_{\mathcal{R}^{*}\left(S^{2} \backslash K_{j}\right)^{[i]}}$. Thus the restriction $H_{j}$ of $H$ on $\mathcal{R}^{*}\left(S^{2} \backslash K_{j}\right)^{[i]}$ is also a Hamiltonian function with respect to the restricted symplectic structure $\omega_{j}$. We have $H_{1}\left(\phi_{\beta}\left(\mu_{1}\right), \cdot\right)=$ $H_{2}\left(\phi_{\beta}\left(\mu_{2}\right), \cdot\right)$ and $H_{j}(x, s)=H_{j}\left(\phi_{\beta_{j}}(x), s+1\right)$. Both $H_{1}$ and $H_{2}$ agree smoothly on the identified meridian $\mu_{1}$ and $\mu_{2}$. So we denote by $H=H_{1} \star H_{2}$. Every Hamiltonian function on $\mathcal{R}^{*}\left(S^{2} \backslash K\right)^{[i]}$ can be expressed in such a way. 
Recall that the group operation on the space of Hamiltonians is given by $H \circ H^{\prime}=$ $H(x, s)+H^{\prime}\left(\left(\phi_{H}^{s}\right)^{-1}(x), s\right)$ and the inverse $H^{-1}$ of $H$ under this operation is $H^{-1}(x, s)=$ $-H\left(\left(\phi_{H}^{s}\right)(x), s\right)$. So $H \circ H^{-1}=0$. Such an operation corresponds to the composition of the induced symplectic diffeomorphisms on the symplectic manifold. We need to construct a cobordism between the moduli spaces with fixed asymptotic values. This is why we use this operation $H \circ H^{\prime}$ in our construction.

Lemma 4.4. Over a monotone symplectic manifold $(M, \omega)$, the zero dimensional moduli space $\mathcal{M}_{J}^{0}(x, x)$ of J-holomorphic curves consists of an isolated constant flow $\{u(t)=x\}$.

Proof: By the monotonicity, the symplectic action of any $J$-holomorphic curves $u \in$ $\mathcal{M}_{J}^{0}(x, x)$ is zero. There are no nonconstant $J$-holomorphic curves in $(M, \omega)$.

For the zero dimensional moduli space $\mathcal{M}_{J_{2}, H_{2}}^{0}\left(\rho_{2}, \rho_{2}\right)$ on the monotone symplectic manifold $\left(\mathcal{R}^{*}\left(S^{2} \backslash K_{2}\right)^{[i]}, \omega_{2}\right)$, we have an one parameter family of $J$-holomorphic curves

$$
\bar{\partial}_{J_{2}, H_{2, \tau}} u=\frac{\partial u}{\partial t}+J_{2}\left(\frac{\partial u}{\partial s}-X_{2, s}^{\tau}(u)\right)=0,
$$

where $H_{2, \tau}=H_{2} \circ \tau H_{2}^{-1}$ for $\tau \in[0,1]$, and $X_{2, s}^{\tau}$ is the corresponding Hamiltonian vector field. Such a family preserves the fixed point $\rho_{2}$ at end, and at $\tau=0$ gives the element in $\mathcal{M}_{J_{2}, H_{2}}^{0}\left(\rho_{2}, \rho_{2}\right)$, and at $\tau=1$ produces a unique element in $\mathcal{M}_{J_{2}}^{0}\left(\rho_{2}, \rho_{2}\right)=\left\{\rho_{2}\right\}$ by Lemma 4.4. So the moduli space $\bar{\partial}_{J_{2}, H_{2, \tau}}^{-1}(0)$ is regular at ends $\tau=0$ and $\tau=1$, and the projection $\pi: \cup_{\tau \in[0,1]} \bar{\partial}_{J_{2}, H_{2, \tau}}^{-1}(0) \rightarrow[0,1]$ is transverse at $\tau$. So

$$
\# \mathcal{M}_{J_{2}, H_{2}}^{0}\left(\rho_{2}, \rho_{2}\right)=\# \mathcal{M}_{J_{2}, 0}^{0}\left(\rho_{2}, \rho_{2}\right)= \pm 1
$$

by the unique constant trajectory flow $\left\{\rho_{2}\right\}$ in Lemma 4.4.

Proposition 4.5. For $\rho_{1} \star \rho_{2}$ and $\rho_{1}^{\prime} \star \rho_{2}$ in $\mathcal{R}^{*}\left(S^{3} \backslash K\right)^{[i]}$ with $\mu_{K}\left(\rho_{1} \star \rho_{2}, \rho_{1}^{\prime} \star \rho_{2}\right) \equiv 1$ $(\bmod 2 N(K))$, the one dimensional moduli space $\mathcal{M}_{J, H}^{1}\left(\rho_{1} \star \rho_{2}, \rho_{1}^{\prime} \star \rho_{2}\right)$ has the following property:

$$
\# \hat{\mathcal{M}}_{J, H}^{1}\left(\rho_{1} \star \rho_{2}, \rho_{1}^{\prime} \star \rho_{2}\right)=\# \hat{\mathcal{M}}_{J_{1}, H_{1}}^{1}\left(\rho_{1}, \rho_{1}^{\prime}\right),
$$

where $\# \hat{\mathcal{M}}_{J, H}^{1}\left(\rho_{1} \star \rho_{2}, \rho_{1}^{\prime} \star \rho_{2}\right)$ is the algebraic number of the zero dimensional manifold $\hat{\mathcal{M}}_{J, H}^{1}\left(\rho_{1} \star \rho_{2}, \rho_{1}^{\prime} \star \rho_{2}\right)$.

Proof: We consider the bundle of Banach spaces $\mathcal{E}^{0,1} \rightarrow L_{1}^{p}\left(\operatorname{Map}\left(\mathbb{C}, \mathcal{R}^{*}\left(S^{2} \backslash K\right)^{[i]}\right)\right)=B$ and the section $\bar{\partial}_{J, H}$. We take the balanced maps for elements in $B$ with asymptotic values $\rho_{1} \star \rho_{2}$ and $\rho_{1}^{\prime} \star \rho_{2}$. Denote by $\hat{B}\left(\rho_{1} \star \rho_{2}, \rho_{1}^{\prime} \star \rho_{2}\right)$. The section $\bar{\partial}_{J, H}$ is $t$-invariant and $\bar{\partial}_{J, H}$ induces a section on the quotient bundle $\hat{\mathcal{E}}^{0,1} \rightarrow \hat{B}\left(\rho_{1} \star \rho_{2}, \rho_{1}^{\prime} \star \rho_{2}\right)$, where $H=H_{1} \star H_{2}$. The fiber of $\hat{\mathcal{E}}^{0,1}$ is given by $L^{p}\left(T^{0,1} \mathbb{C} \otimes_{J} u^{*} T \mathcal{R}^{*}\left(S^{2} \backslash K\right)^{[i]}\right)$ with $\int_{-\infty}^{0}|\nabla u|^{2}=\int_{0}^{\infty}|\nabla u|^{2}$ for $u \in \hat{B}\left(\rho_{1} \star \rho_{2}, \rho_{1}^{\prime} \star \rho_{2}\right)$. Again the zero set of $\bar{\partial}_{J, H}$ is a zero dimensional manifold $\hat{\mathcal{M}}_{J, H}^{1}\left(\rho_{1} \star \rho_{2}, \rho_{1}^{\prime} \star \rho_{2}\right)$ inside $\hat{B}\left(\rho_{1} \star \rho_{2}, \rho_{1}^{\prime} \star \rho_{2}\right)$ for the one dimensional component $\hat{\mathcal{M}}_{J, H}^{1}\left(\rho_{1} \star\right.$ 
$\left.\rho_{2}, \rho_{1}^{\prime} \star \rho_{2}\right)$. The orientation at each point in $\hat{\mathcal{M}}_{J, H}^{1}\left(\rho_{1} \star \rho_{2}, \rho_{1}^{\prime} \star \rho_{2}\right)$ is the orientation on the trajectory flow used in defining the symplectic Floer boundary. By the monotonicity and the Floer-Gromov compactness,

$$
\# \hat{\mathcal{M}}_{J, H}^{1}\left(\rho_{1} \star \rho_{2}, \rho_{1}^{\prime} \star \rho_{2}\right)=\# \bar{\partial}_{J, H}{ }^{-1}(0)
$$

is well-defined.

Let $\tau \in[0,1]$ and $\bar{\partial}_{J, H_{\tau}}$ be a section on $\hat{\mathcal{E}}^{0,1} \rightarrow \hat{B}\left(\rho_{1} \star \rho_{2}, \rho_{1}^{\prime} \star \rho_{2}\right)$, where $H_{\tau}=H_{1} \star\left(H_{2} \circ\right.$ $\left.\tau H_{2}^{-1}\right)$. For each $\tau$ the section $\bar{\partial}_{J, H_{\tau}}$ has the transverse zeros by [6]. For $\tau=0$,

$$
\bar{\partial}_{J, H_{0}}^{-1}(0)=\bar{\partial}_{J, H_{1} \star H_{2}}^{-1}(0)=\hat{\mathcal{M}}_{J, H}^{1}\left(\rho_{1} \star \rho_{2}, \rho_{1}^{\prime} \star \rho_{2}\right) .
$$

Let $\hat{\mathcal{M}}_{J, H_{\tau}}^{1}\left(\rho_{1} \star \rho_{2}, \rho_{1}^{\prime} \star \rho_{2}\right)$ be the zeros of the section $\bar{\partial}_{J, H_{\tau}}$ for $\tau \in[0,1]$. For $\tau=1$, the zero set $\bar{\partial}_{J, H_{1} \star\left(H_{2} \circ H_{2}^{-1}\right)}^{-1}(0)=\bar{\partial}_{J, H_{1} \star 0}^{-1}(0)$ becomes solutions of

$$
\frac{\partial u}{\partial t}+J\left(\frac{\partial u}{\partial s}-X_{1, s}(u)\right)=0 .
$$

The $J$-holomorphic curve $u$ of (30) satisfies the property that $u_{1} \star u_{2}$ with $\mu_{K_{1}}\left(u_{1}\right)=1$ and $\mu_{K_{2}}\left(u_{2}\right)=0$. Thus $u_{2} \in \mathcal{M}_{J_{2}, H_{2} \circ H_{2}^{-1}}^{0}\left(\rho_{2}, \rho_{2}\right)$ is the unique element $\left\{\rho_{2}\right\}$ by Lemma 4.4 . Hence $u=u_{1} \star\left\{\rho_{2}\right\}$ can be identified as an element $u_{1}$ in $\hat{\mathcal{M}}_{J_{1}, H_{1}}^{1}\left(\rho_{1}, \rho_{1}^{\prime}\right)$.

Then $\tau=0$ and $\tau=1$ in $[0,1]$ are regular values of the projection $\pi_{1}: \cup_{\tau \in[0,1]} \hat{\mathcal{M}}_{J, H_{\tau}} \rightarrow$ $[0,1]$ since the one dimensional moduli space $\cup_{\tau \in[0,1]} \hat{\mathcal{M}}_{J, H_{\tau}}$ is transverse to $\hat{B}\left(\rho_{1} \star \rho_{2}, \rho_{1}^{\prime} \star \rho_{2}\right)$ (see [5, 6, 14, 24]). So the parameterized moduli space $\cup_{\tau \in[0,1]} \hat{\mathcal{M}}_{J, H_{\tau}}$ is an one dimensional submanifold in the product space $[0,1] \times \hat{B}\left(\rho_{1} \star \rho_{2}, \rho_{1}^{\prime} \star \rho_{2}\right)$ with oriented boundary components $-\hat{\mathcal{M}}_{J, H}^{1}\left(\rho_{1} \star \rho_{2}, \rho_{1}^{\prime} \star \rho_{2}\right)$ and $\hat{\mathcal{M}}_{J_{1}, H_{1}}^{1}\left(\rho_{1}, \rho_{1}^{\prime}\right) \star\left\{\rho_{2}\right\}$. Each boundary component is compact zero dimensional manifold and $\cup_{\tau \in[0,1]} \hat{\mathcal{M}}_{J, H_{\tau}}$ is also compact by the Floer-Gromov compactness theorem. So $\hat{\mathcal{M}}_{J, H}^{1}\left(\rho_{1} \star \rho_{2}, \rho_{1}^{\prime} \star \rho_{2}\right)$ and $\hat{\mathcal{M}}_{J_{1}, H_{1}}^{1}\left(\rho_{1}, \rho_{1}^{\prime}\right) \star\left\{\rho_{2}\right\}$ are oriented cobordant:

$$
\begin{aligned}
0 & =\partial\left(\cup_{\tau \in[0,1]} \hat{\mathcal{M}}_{J, H_{\tau}}\right) \\
& =-\# \bar{\partial}_{J, H_{\tau}=H_{0}}{ }^{-1}(0)+\# \bar{\partial}_{J, H_{\tau}=H_{0}}{ }^{-1}(0) \\
& =-\# \hat{\mathcal{M}}_{J, H}^{1}\left(\rho_{1} \star \rho_{2}, \rho_{1}^{\prime} \star \rho_{2}\right)+\# \hat{\mathcal{M}}_{J_{1}, H_{1}}^{1}\left(\rho_{1}, \rho_{1}^{\prime}\right) \star\left\{\rho_{2}\right\} \\
& =-\# \hat{\mathcal{M}}_{J, H}^{1}\left(\rho_{1} \star \rho_{2}, \rho_{1}^{\prime} \star \rho_{2}\right)+\# \hat{\mathcal{M}}_{J_{1}, H_{1}}^{1}\left(\rho_{1}, \rho_{1}^{\prime}\right),
\end{aligned}
$$

since the orientation of $u_{1} \star\left\{\rho_{2}\right\}$ is compatible with the orientations of $\rho_{1} \star \rho_{2}$ and $\rho_{1}^{\prime} \star \rho_{2}$. Hence the result follows.

Proof of Theorem 4.3: By Corollary 3.5 and Proposition 4.5, we have that all possible one dimensional moduli spaces $\mathcal{M}_{J, H}^{1}\left(\rho_{1} \star \rho_{2}, \rho_{1}^{\prime} \star \rho_{2}^{\prime}\right)$ are given by the asymptotic values same on one of $\mathcal{R}^{*}\left(S^{3} \backslash K_{j}\right)^{[i]}(j=1,2)$ and differ by one Maslov index on the other. There is no nontrivial $J$-holomorphic curve with negative Maslov index on the monotone symplectic 
manifolds. So the first differential $d_{1}$ is given by

$$
d_{1}=\partial_{1}^{\left(r_{1}\right)} \star \operatorname{Id}_{2} \pm \operatorname{Id}_{1} \star \partial_{2}^{\left(r_{2}\right)} \pm d_{\beta_{1}} \star \operatorname{Id}_{2} \pm \operatorname{Id}_{1} \star d_{\beta_{2}}+\delta_{\beta_{1}} \star \operatorname{Id}_{2} \pm \operatorname{Id}_{1} \star \delta_{\beta_{2}},
$$

for all possible $\rho_{1} \star \rho_{2}$ in Proposition 3.1. One needs to check that (24) is indeed a differential, i.e., $d_{1} \circ d_{1}=0$. This follows by straightforward calculation by using the remark after Theorem 4.3 .

Remarks: (i) The identification of $d_{1}$ is highly depending on the monotonicity of $\mathcal{R}^{*}\left(S^{2} \backslash\right.$ $K)^{[i]}$. By [6] and Proposition 3.4, we reduce the problem to the moduli space with special

asymptotic values (one of $\rho_{j}$ and $\rho_{j}^{\prime}(j=1,2)$ agrees). Then we construct a cobordism to compute the first differential $d_{1}$.

(ii) In [16, 18], we studied the structure of all moduli spaces with index $\leq 4$ by a gluing result. Using certain cohomology classes, we identified all the higher differentials in [18. In order to identify $d_{2}$ in Theorem 3.9, we need to get the local structure of $J$-holomorphic curves on $\mathcal{R}^{*}\left(S^{2} \backslash K\right)^{[i]}$ which is similar to the gluing result in [16], and then find the correct cohomology class of degree one for the differential $d_{2}$. We will discuss this elsewhere.

(iii) Note that $\mathcal{R}^{*}\left(S^{2} \backslash K\right)$ can be identified as a symplectic fiber product of $\mathcal{R}^{*}\left(S^{2} \backslash K_{1}\right)$ and $\mathcal{R}^{*}\left(S^{2} \backslash K_{2}\right)$. We hope that our discussion in this paper may shed a light on the study of the symplectic Floer homology of the fiber product of two symplectic manifolds.

\section{REFERENCES}

[1] J. S. Birman, Braids, links and mapping class groups, Ann. Math. Studies, No. 82, Princeton University Press (1974).

[2] R. Bott, Nondegenerate critical manifolds, Ann. Math. 60(1954), 248-261.

[3] S. Cappell, R. Lee and E. Miller, Equivariant Casson invariant, preprint.

[4] S. Cappell, R. Lee and E. Miller, On the Maslov index, Comm. Pure. Appl. Math, Vol XLVII, 121-186 (1994).

[5] S. K. Donaldson and P. B. Kronheimer, The Geometry of Four-manifolds, Oxford Mathematical Monographs, Oxford University Press, Oxford, 1990.

[6] A. Floer, Symplectic fixed points and holomorphic spheres, Comm. Math. Phys. 120, 575-611 (1989).

[7] A. Floer, An instanton invariant for 3-manifolds, Commun. Math. Phys. 118 (1988), 215-240.

[8] A. Floer and H. Hofer, Coherent orientations for periodic orbit problems in symplectic geometry, Math. Z., 212, 13-38 (1993).

[9] R. Fintushel, R. Stern, Integer graded instanton homology groups for homology three spheres, Topology, 31: 589-604, (1992).

[10] M. Gromov, Pseudoholomorphic curves in symplectic manifolds, Invent. Math. 82, 307-347, (1985).

[11] C. Herald, Legendrian cobordism and Chern-Simons Theory on 3-manifolds with boundary, Comm. Anal and Geom., 2, No.3 (1994), 337-413.

[12] C. Herald, Flat connections, the Alexander invariant and Casson's invariant, Comm. Anal. Geom. 5 (1997), no.1, 93-120.

[13] E. Klassen, Representations of knot groups in SU(2), Trans. AMS, Vol 326, No 2, 795-828 (1991).

[14] Lê Hông Vân and K. Ono, Symplectic fixed points, the Calabi invariant and Novikov homology, Topology, Vol 34, No 1. 155-176 (1995).

[15] W. Li, Casson-Lin's invariant and Floer homology, J. Knot Theory and its Ramification, Vol 6, No.6 (1997), 851-877.

[16] W. Li, Floer homology for connected sums of homology 3-spheres, J. Diff. Geom, 40, 129-154 (1994). 
[17] W. Li, Equivariant knot signatures and Floer homologies, OSU preprint (1997) (submitted).

[18] W. Li, Connected sums, Floer homologies and spectral sequences, OSU preprint (1996) (submitted).

[19] W. Li, Künneth formulae and cross products for the symplectic Floer cohomology, OSU preprint (1997) (submitted).

[20] X. S. Lin, A knot invariant via representation spaces, J. Diff. Geom., 35, 337 - 357 (1992).

[21] J. Milnor, Construction of Universal bundles, I, Annals of Math. Vol 63, No. 2, 272-284, (1956).

[22] Y. Oh, Floer cohomology, spectral sequences, and the Maslov class of Lagrangian embeddings, IMRN, No. 7 (1996), 305-346.

[23] D. Rolfsen: Knots and Links, Publish or Perish, Inc, (1976, 1990).

[24] D. Salamon and E. Zehnder, Morse theory for periodic solutions of Hamiltonian systems and the Maslov index, Comm. Pure Appl. Math. 45 (1992), no. 10, 1303-1360.

[25] S. Smale, Diffeomorphisms of the 2-sphere, Proc. AMS, 10(1959), 621-626.

[26] E. Spainier, Algebraic Topology, McGraw-Hill, New York, 1966.

Department of Mathematics

Oklahoma State University

StillWATER, OKLAHOMA 74078-0613

U. S. A.

E-mail address: wli@littlewood.math.okstate.edu 